\title{
Acknowledgement to Reviewers of International Journal of Molecular Sciences in 2015
}

\author{
International Journal of Molecular Sciences Editorial Office
}

Published: 21 January 2016

MDPI AG, Klybeckstrasse 64, CH-4057 Basel, Switzerland; ijms@mdpi.com

The editors of International Journal of Molecular Sciences would like to express their sincere gratitude to the following reviewers for assessing manuscripts in 2015.

We greatly appreciate the contribution of expert reviewers, which is crucial to the journal's editorial decision-making process. Several steps have been taken in 2015 to thank and acknowledge reviewers. Good, timely reviews are rewarded with a discount off their next MDPI publication. By creating an account on the submission system, reviewers can access details of their past reviews, see the comments of other reviewers, and download a letter of acknowledgement for their records. In addition, MDPI has launched a collaboration with Publons, a website that seeks to publicly acknowledge reviewers on a per journal basis. This is all done, of course, within the constraints of reviewer confidentiality. Feedback from reviewers shows that most see their task as a voluntary and mostly unseen work in service to the scientific community. We are grateful to our reviewers for the contribution they make.

\author{
Abbiati, Giorgio \\ Abbott, Karen L. \\ Abbt-Braun, Gudrun \\ Abdallah, Chadi G. \\ Abdel-Mottaleb, M.M.A. \\ Abenavoli, Ludovico \\ Abend, Michael \\ Abou Neel, Ensanya A. \\ Abraham, Solana \\ Abusco, Anna Scotto \\ Acién Fernández, F.G. \\ Acord, John \\ Acuña, Darío \\ Adamakis, I.-D.S. \\ Adams, James B. \\ Adler, Carrie \\ Adriano, Marocco \\ Afantitis, Antreas \\ Afolayan, A.J. \\ Agostini, Marco \\ Agostino, Mark \\ Agoston, Denes V. \\ Aharoni, Rina \\ Ahlenstiel, Golo \\ Ahmad, Faiz U. \\ Ahmed, Hafiz \\ Ahn, Sang-hoon
}

Hoffman, Heinrich
Hoffman, Steven A.
Hoffmann, Klaus
Hoffmann, Markus H.
Hoffmann, Mathias
Höfinger, Siegfried
Hofmann, Johann
Hogan, Andrew
Hohenegger, Martin
Hojjat-Farsangi, M.
Holden, Patricia
Holding, David R.
Holen, Ingunn
Holick, Michael F.
Holland, Peter W.H.
Hollander, W. den
Hollenstein, Marcel
Holliday, Dawn
Holman, Theodore
Holmberg, Scott D.
Holmes, Robert
Holmstrøm, Kim
Holst, Jens Juul
Höltinger, Stefan
Hondan, Tomoyuki
Hong, Mee Young
Hong, Yonggeun

Pelkonen, Olavi

Pellegrini-Giampietro, D.E.

Pelletier, Stephane

Pelosi, Paolo

Pena, Romi N.

Penders, Thomas M.

Penfold, Scott N.

Penfornis, Patrice

Peng, Wen-Huang

Peng, Zhenling

Pennypacker, Keith

Penzes, Peter

Pepys, Mark

Peral, Belén

Perales, Oscar

Perego, Carla

Pereira, C.S.A.

Pereira, David

Pereira, Florbela

Pereira, Leonel

Pereira, Olívia R.

Perepelyuk, Maryna

Peretz, Avi

Pérez, Emilio M.

Pérez, Montse

Perez-Cano, Francisco

Pérez-Castrillón, José L. 
Aikawa, Shimpei

Aikawa, Takuya

Ainscough, Justin F.X.

Akazawa, Chihiro

Akbar, S.M.F.

Akbari, Mansour

Akbari, Mohammad R.

Akbarzadeh, A.H.

Akeda, Koji

Akita, Sadanori

Akopian, Armen N.

Al Ghouleh, Imad

Alabugin, Igor

Al-Ahmadie, Hikmat A.

Alavez, Silvestre

Alavi, Saman

Albalat, Amaya

Albero, Cenci

Alcoutlabi, Mataz

Al-Ejeh, Fares

Aleman, Soo

Alessandro, Passera

Alevizos, Ilias

Alexeyev, Mikhail

Alexiou, Christoph

Alexis, Frank

Alfaidy, N.

Alfandari, Dominique

Alfonso, Ignacio

Alfonso, Miguel

Alfonzo, Antonio

Alikhani, Esmail

Alison, Malcolm R.

Alkan, Noam

Al-Kateb, Hussam

Allahverdiyeva, Yagut

Allain, Jean-Pierre

Allinson, Sarah L.

Almasio, P.L.

Alonso, Maria Carmen

Aloulou, Ahmed

Alpini, Gianfranco

Altarawneh, M.

Altenbach, Susan B.

Alvarez-Suarez, J.M.

Aly, Hamed

Alzaid, F.

Amado, Francisco

Amarasinghe, V.

Amarenco, Gerard

Amatngalim, Gimano D.

Ambs, Stefan

Ami, Diletta
Honoré, Patrick M.

Hool, Livia C.

Hordvik, Ivar

Horii, Takuro

Horn, R.

Horovitz, Amnon

Hörsch, Dieter

Hortelano, Sonsoles

Horvat, Josip

Horvath, David P.

Hossein-Babaei, Faraz

Hou, Chien-Wei

Hou, Jianghui

Hou, Susan

Houde, Mario

Houee-Levin, Chantal

Houng, Jer-yiing

Houry, Walid A.

Houshmand, Massoud

Houston, Kevin D.

Houston, Mark C.

Houston, Ross

Howell, Tyson

Howell, Viive

Hoyer-Fender, Sigrid

Hsia, Shih-Min

Hsieh, Chung-Bao

Hsieh, Yi-Hsien

Hsu, Chin-lin

Hsu, Chung Y.

Hsu, Fu-yin

Hsu, Hsue-Yin

$\mathrm{Hsu}, \mathrm{Tc}$

Hsueh, Yi-Huang

$\mathrm{Hu}, \mathrm{Bo}$

$\mathrm{Hu}$, MingKuan

$\mathrm{Hu}$, Valerie W.

$\mathrm{Hu}$, Zhuowei

Huang, Chiu-jung

Huang, Chi-Yu

Huang, Chun-Jung

Huang, Fei

Huang, Feihe

Huang, Hsuan-Cheng

Huang, Jiwei

Huang, Li-Fen

Huang, Peng

Huang, Shih-ming

Huang, Shuang

Huang, Tim Hui-Ming

Huang, Wen-Li

Huang, Ya-ling

Huang, Yi-Wen
Perez-Jimenez, Raul

Pérez-Sánchez, Jaume

Périgo, E.A.

Perks, Claire M.

Perl, Andras

Perona, John J.

Perret, Xavier

Perricone, Carlo

Perrier, Aurélie

Perrine-Walker, F.M.

Perron, Karl

Persoz, Charles

Persson, Kenneth

Persson, Rasmus

Perumal, Deepak

Peruzzi, Francesca

Peterman, Erwin J.G.

Peters, Rene

Petersen, Christian P.

Petersen, Elijah

Peterson, Julia

Petrlova, Jitka

Pezzotti, Giuseppe

Pflugmacher, Stephan

Phillips, Jacqueline K.

Philpott, Mike

Pi, Liya

Piazza, Fabrizio

Piccialli, Gennaro

Piccinini, Anna

Piccoli, G.B.

Pichat, Pierre

Pichler, Martin

Pickkers, Peter

Picklo, Matthew J.

Piga, Matteo

Piggins, Hugh D.

Pignata, Claudio

Pihlanto, Anne

Pillay, Viness

Pillon, Yohan

Pilmé, Julien

Pilon, Marc

Pilon-Thomas, Shari

Pinalli, Roberta

Pincus, Seth H.

Pino-Figueroa, Alejandro

Pinton, Paolo

Pippucci, Tommaso

Pires, J.C.M.

Pirillo, Angela

Pirola, Carlos

Pitchiaya, S. 
Amira, Zarrouk

Ammendola, Rosario

Amodio, Nicola

Amorati, Riccardo

Amorós, Asunción

An, Wonsuk

Anastasi, Emanuela

Anchan, Raymond

Anders, Hans-joachim

Andersen, M.-B.S.

Andersen, Oluf

Andersen, Sigve

Anderson, Anne J.

Anderson, Kurt

Anderson, Peter D.

Andersson, Jan

Andjelkovic, Anuska V.

Andrade, Paula

Andreadou, Ioanna

Andreana, Peter R.

Angeli, Celestino

Angelico, Francesco

Angelov, Doychin N.

Anglani, Franca

Anifandis, Georgios M.

Anraku, Makoto

Antoniadi, Ioanna

Antonio, Baltazar

Aoki, Kazuhiro

Aoki, Yoshinori

Aparicio, Frederic

Apone, Fabio

Appel, Jens

Appenroth, Klaus

Apte, Swapna

Araki, Wataru

Aravindan, Natarajan

Arbona, Vicent

Arbones-Mainar, Jose

Archer, Trevor

Ardell, David

Arden, Catherine

Argon, Yair

Argyriou, Notis

Ariani, Andrea

Ariga, Katsuhiko

Ariño, Cristina

Arinzeh, T.L.

Arita, Minetaro

Ariunbaatar, Javkhlan

Ariyama, Kaoru

Arkudas, Andreas

Armero Ibáñez, Eva
Huang, Yu

Huber, Veronica

Huchzermeyer, Bernhard

Hull, Joe

Hulten, Edward

Humblot, Vincent

Hunter, Christopher A.

Huppertz, Berthold

Hur, Yoonkang

Hura, Tomasz

Hurlé, Juan

Hussain, M Mahmood

Hussein, Malik

Hust, Michael

Hvidsten, Torgeir R.

Hwa, John

Hwang, Daniel H.

Hwang, David

Hwang, Kuo-jen

Hwang, Ming-Jing

Hwang, Sun Wook

Hwang, Yoon Jung

Hyeon, Changbong

Hynes, Kim

Hyun, Kyung-Yae

Iagaru, Andrei

Ianni, Federica

Iasevoli, Felice

Ibarra Molero, Beatriz

Ibekwe, Mark

Ibrahim, A.G.-E.

Ibrahim, Salam A.

Ichikawa, Hiroshi

Idnurm, Alexander

Ido, Akio

Idoyaga, Juliana

Ientile, Riccardo

Iglesias, Domingo J.

Iglesias-Fernández, R.

Iguchi, Eriko

Ihara, Eiji

Ikari, Yuji

Ikawa, Masahito

Ikeda, Masahiro.

Ilan, Ezgi

Illing, Robert-Benjamin

Im, Hee-Jeong

Imai, Hirotaka

Imaizumi, Kazunori

Imataka, Hiroaki

Immel, Françoise

Imokawa, Genji

Imumorin, Ikhide G.
Pittelkow, Michael

Pittman, Jon K.

Pitto, Letizia

Piva, Terrence

Platt, Jeffrey L.

Platts, James

Plett, Jonathan

Pliego-Alfaro, Fernando

Plourde, Guy

Poblaciones, Maria J.

Poerschmann, Juergen

Pognonec, Philippe

Pointillart, Fabrice

Poirier, Isabelle

Poldermans, Don

Polerecky, Lubos

Polesskaya, Anna

Polishchuk, Roman

Pollastri, Gianluca

Pollmann, Katrin

Poma, Alessandro

Pomar, Federico

Pomero, Fulvio

Poncet, Valérie

Ponchon, Luc

Ponomarev, Eugene D.

Ponsaerts, Peter

Pontaño, Francisco Pérez

Pook, Mark A.

Popa-Wagner, Aurel

Poptani, Harish

Pörtner, Ralf

Posada Estefan, Olga M.

Possee, Robert

Potaczek, Daniel P.

Pouli, Nicole

Poulos, Thomas L.

Powell, J.T.

Powell, N.

Powers, Robert

Pradhan, Sriharsa

Prado, Rafael de

Prasad, Sadheo

Prasanth, K.V.

Prashanth, Konda G.

Prat, Maria

Preissner, Klaus T.

Prentis, Peter J.

Proestos, Charalampos

Proust, Hélène

Prudovsky, Igor

Prywes, Ron

Pucker, Andrew D. 
Armstrong, Ehrin J.

arnhold, juergen

Aroca, Ricardo

Artlip, Timothy

Arugula, Mary

Aruoma, Okezie

Asahi, Michio

Asakura, Tomiko

Asano, Koichiro

Asatryan, Liana

Ashfaq, M.K.

Ashraf, Shahid

Askalan, Rand

Askarian-Amiri, M.E.

Aslam, Muhammad

Assfeld, Xavier

Asterholm, I.W.

Atanassova, Rossitza

Atangana, Alain R.

Atef, Eman

Atkins, John F.

Atomi, Haruyuki

Atrian, Silvia

Atsukawa, Masanori

Attardo, Geoffrey M.

Audebert, Marc

Audenaert, Kris

Auer, Manfred

Auerbach, Sanford

Auguet, Teresa

Aureliano, M.

Aurora, Rajeev

Austin, Brian

Avarre, Jean-Christophe

Avila, Matias A.

Avola, R.

Avram, Anca M.

Avvedimento, Enrico V.

Ayala-Zavala, J.F.

Ayalew, Sahlu

Ayonrinde, Oyekoya T.

Azad, Arun

Azimzadeh, Omid

Azmi, Asfar

Azorín, Fernando

Azuma, Kazuo

Azuma, Toshifumi

Baba, Hideo

Babbs, Charles F.

Babiak, Igor

Babiuk, Shawn

Bach, Horacio

Bache, Søren
Indolfi, Giuseppe

Indriolo, Emily

Infascelli, $\mathrm{F}$.

Inoue, Keiji

Inselman, Amy

Insenser, María

Inui, Masafumi

Inukai, Yoshiaki

Inuzuka, Hiroyuki

Invernizzi, Pietro

Ioelovich, Michael

Irato, Paola

Isani, Gloria

Ishaque, Ali

Ishibashi, Kei

Ishii, Tetsuya

Ishijima, Sumio

Ishikawa, Yasuko

Ishizaki, Takuma

Ishmael, Jane E.

Isidori, Andrea M.

Isobe, Naoki

Isokpehi, Raphael D.

Isshiki, Yoshiaki

Itahana, Koji

Itahashi, Takashi

Ito, Daisuke

Ito, Michiho

Ito, Takuya

Ito, Yasuhiro

Itoh, Toshiyuki

Itoh, Yoshifumi

Iughetti, Lorenzo

Ivan, Rajkovic

Ivanov, A.G.

Ivessa, N. Erwin

Ivkov, Robert

Iwaizumi, Masakazu G.

Iwamori, Masao

Iwamoto, Sadahiko

Iwasaki, Kenta

Iwasaki, Tomohiro

Iwata, Junichi

Izawa, Hironori

Izumiya, Yasuhiro

Jacob, Francis

Jacob, Melissa

Jacobsen, E.E.

Jadhav, Vaibhav

Jahandideh, Samad

Jaime, Laura

Jaki, Birgit

Jakočiūnas, Tadas
Puddu, Paolo Emilio

Puentes, Fabiola

Puértolas, Eduardo

Puglisi, Edoardo

Puinean, Alin M.

Pulford, Karen

Puppo, Francesco

Putz, Mihai V.

Qi, Qungang

Qi, Yiping

Qian, Hai

Qian, Li

Qian, Pengxu

Qian, Wei

Qian, Yanrong

Qiao, Kun

Qin, Yang

Quan, Guoxing

Queiroga, Cláudia S.F.

Quesada, Víctor

Quintana, José

Quirino, Rafael

Quistorff, Bjørn

Quock, Ryan L.

Rabbani, Naila

Rabilloud, F.

Rachmawati, Dessy

Radović, Branislav

Raedschelders, Koen

Raffaele, Sylvain

Raftery, Martin

Ragni, Laura

Ragoussis, Jiannis

Ragozin, Igor

Ragusa, Maria Antonietta

Ragusa, Santo

Rahier, Hubert

Rahmani, Mohamed

Rajakaruna, Nishanta

Rajam, Gowrisankar

Rajaputra, Pallavi

Rajendran, Praveen

Rajput, Nav

Rak, Janusz

Ramachandran, Girish

Ramakrishnan, S.

Raman, A.

Ramaraj, Pandurangan

Ramchandran, Lata

Ramos, Javier

Ramos, Sonia

Ramos-Solano, Beatriz

Ramsden, Lawrence 
Bachmair, Andreas

Backström, Tobias

Badylak, S.F.

Bagby, Michael

Bagchi, Anindya

Bagnaninchi, Pierre

Bagnato, Alessandro

Bagos, Pantelis

Bai, Shuhua

Bailly, Sabine

Bajinskis, Ainars

Baker, Mark D.

Bakkeren, Guus

Balabaud, Charles

Balajee, Adayabalam

Balasubramanian, Meena

Balaz, Milan

Baldessarini, Ross J.

Balduini, Walter

Baleja, Jim

Balemba, Onesmo B.

Balestrini, Raffaella

Balietti, Marta

Ball, Eldon

Ballestri, Stefano

Ballizany, Wouter

Ballmer-Hofer, Kurt

Baltazar, Fátima

Baluska, Frantisek

Bamdad, Cynthia

Bandarra, Narcisa

Banerjee, Hirendra nath

Banerjee, Sanjeev

Banerjee, Taraswi

Baniahmad, Aria

Banilas, Georgios

Bannwarth, Markus B.

Bantel, Carsten

Bao, Yuping

Bao, Zhilong

Bapputty, Reena

Baraka, Khaled

Barakat, Abdellatif

Baranski, Matthew

Baranzini, Sergio E.

Barash, Itamar

Barbarossa, I.T.

Barbera, Mariagnese

Barberi, Tiziano

Barberon, Marie

Barbier, François F.

Barbu, Eugen

Barbucci, Rolando
Jakubowski, Hieronim

Jalili, Thunder

James, Aaron

Jameson, David

Jamieson, Stephen M.F.

Jan, Eric

Jan, Tong Rong

Jandrić, Z.

Janesko, Benjamin G.

Jang, Byoung Kuk

Jang, Mi-Kyeong

Jang, Seonghoe

Janitz, Michael

Janji, Bassam

Jansen, Andrew N.

Jansen, Gerrit

Jarret, Robert L.

Jat, Parmjit S.

Javed, Fawad

Jaworska, Natalia

Jayasinghe, Suwan

jean, Ludovic

Jeanmart, Stephane

Jefferies, L.K.

Jeffery, Constance J.

Jeffery, Glen

Jeffree, Rosalind L.

Jellinger, Kurt

Jemli, Sonia

Jenkins, Jill A.

Jensen, Eric

Jensen, Kenneth

Jensen, L.H.

Jensen, Thomas

Jeon, Tae-Joon

Jeong, Daewon

Jernigan, Nikki L.

Jeschke, Udo

Jessel, Nadia

Ji, Debin

Ji, Yingbiao

Jia, Chenxi

Jia, Zongchao

Jiang, Shu-Ye

Jimi, Eijiro

Jin, Chunyang

Jin, Takashi

Jing, Fuyuan

Jobling, Malcolm

Johann, Christoph

Johannsen, Darcy

Johnson, Hope A.

Johnson, Jason L.
Rana, D.

Randall, Stephen K.

Randazzo, Antonio

Rangan, Vijaya

Rangasamy, Jaykumar

Ranger, Christopher M.

Ranty, Benoit

Rapposelli, Simona

Ratcliffe, George

Rathbone, Alex J.

Rathinavelu, Appu

Ratner, Nancy

Rattray, Ben

Rau, Julietta V.

Rauch, Bernhard

Rauch, U.

Rauniyar, Navin

Raut, Sangram

Ravegnini, Gloria

Ray, Ratna

Ray, Udayan

Rayburn, Keith

Raychaudhuri, Pradip

Re, Suyong

Reading, Benjamin J.

Real, María Dolores

Reale, Marcella

Reason, Donald

Rebe, Cedric

Rebhun, Robert

Rebl, Alexander

Rebmann, V.

Reboll, Marc R.

Reche, Pedro A.

Reddy, Ashvini K.

Reddy, M.V.

Reddy, Umesh K.

Redfern, Julie

Ree, Kim Mee

Reeve, Lesley

Reeve, Vivienne

Rehder, Dieter

Rehwinkel, Jan

Reich, Stephen

Reichel, Martin

Reimers, Kerstin

Reinach, Peter S.

Reis, Flávio

Reiser, Peter J.

Reisman, Scott A.

Reissmann, Siegmund

Remans, Tony

Remington, Gary 
Barbuti, Andrea

Barcaccia, Gianni

Barcellos-de-Souza, Pedro

Barcia, Jorge M.

Bard, Frederic

Bardoni, Barbara

Barea, E.

Barhoum, Ahmed

Barlow, Linda

Barnea, Eytan R.

Barnes, D.W.

Barny, Marie-Anne

Barolo, Claudia

Barr, Stephen D.

Barrajón-Catalán, Enrique

Barrangou, Rodolphe

Barreira, João

Barresi, Valeria

Barreto, Maria Do Carmo

Barrientos, Antoni

Barroso, Gérard

Bartali, R.

Bartfeld, Sina

Bartoloni, Giovanni

Bartscherer, Kerstin

Barzilay, Joshua I.

Basaraba, Randall J.

Baschant, Ulrike

Basini, Giuseppina

Bastola, Dhundy R.

Bastolla, Ugo

Bates, Shannon M.

Batra, Jyotsna

Batra, Surinder

Battaglia, Agatino

Battino, Maurizio

Bauer, Johann

Baum, Christel

Baum, Michel

Baumann, Heinz

Baumgartner, Adi

Bavro, Vassiliy

Bayliss, Sion

Bazhin, Alexandr

Beasley, Shea

Beaton, Lindsay A.

Beckel, J.M.

Becker, Hubert Dominique

Beckmann, Manfred

Bédard, P.-A.

Beggs, Simon

Beier, Justus P.

Bejai, Sarosh
Johnson, Margaret E.

Johnston, Michael

Johny, Manu Ben

Jolivet, Renaud

Jones, Eleanor R.

Jones, John

Jones, T Bucky

Jonesa, G.T.

Jong, Nancy N.

Jonkers, Daisy

Joo, Hong-gu

José, Berna Canovas

Joseph, Jacob

Joshee, N.

Joshi, Nikhil

Jouline, Igor B.

Jovanović, Aleksandar

Joven, Jorge

Juang, Shin-hun

Juarranz, Ángeles

Julian, Borejdo

Julier, Bernadette

Junaid, Mohammed A.

Jung, Chol-Hee

Jung, Ki-Hong

Jung, Klaus

Jung, Kyung Hwan

Jung, Seunho

Jung, Youngmi

Jurk, Diana

Just, Steffen

Juszczak, Lesław

Juzeniene, Asta

Kabbaj, Mohamed

Kaddurah-Daouk, Rima

Kadokawa, Jun-ichi

Kadota, Kazunori

Kagadis, George C.

Kaguni, Jon M.

Kaiser, Brent N.

Kaiser, Jean-pierre

Kajiya, Hiroshi

Kakisis, John D.

Kakulas, Foteini

Kalab, Petr

Kalajdzic, Predrag

Kalendar, Ruslan

Kalinin, Stanislav

Kalinowski, Jörn

Kallenbach, Neville

Kalpaxis, Dimitrios L.

Kaludercic, Nina

Kalve, Shweta
Rempel, L.A.

Ren, Jian-guo

Ren, Xuefang

Renaud, Justine

Renaudineau, Yves

Rengo, Giuseppe

Renner, Bertold

Renner, Ulrich

Renshaw, Stephen A.

Repp, Jascha

Rescifina, Antonio

Ress, Anna Lena

Revilla, Eugenio

Rey, Pascal

Reyes, Fernando

Reyes, José C.

Reygaert, Wanda

Reynolds, Clare M.

Rezaei-Ghaleh, Nasrollah

Rezzani, Rita

Rhee, Dong-Kwon

Ribeiro, Martha S.

Ricciardelli, Carmela

Richardson, Alan

Riddiford, Lynn M.

Rider, Lisa G.

Rigano, Daniela

Rigano, Dott Daniela

Rimessi, Alessandro

Rimondini, Lia

Ringli, Christoph

Riou, Laurent M.

Rishi, Arun K.

Rissiek, Björn

Ris-Stalpers, Carrie

Risueño, María-Carmen

Ritte, Jenna C. Sullivan

Rittner, Heike

Rittschof, Daniel

Riva, Paola

Rivas, Elena

Rivera, Victor

Rivero, Rosa M.

Rizzoti, Karine

Ro, Hyeon Su

Robert, Claude

Roberto, Dominique

Roberts, Ashley

Roberts, Christopher J.

Roberts, Craig W.

Robertson, Mark J.

Robinson, S.M.

Roccheri, Maria Carmela 
Bejan, Iustinian

Bejarano, Ignacio

Belke, Darrell D.

Bellastella, Giuseppe

Bellezza, Ilaria

Bellincampi, Daniela

Belling, Kirstine C.

Bello, Nicholas T.

Benavente-Babace, A

Bendahhou, Saïd

Benetti, Federico

Benfenati, Emilio

Benitah, Jean-Pierre

Benito, Juan M.

Benn, Peter

Bennet, Andrew

Bennink, Martin

Benson, Al B.

Benson, Michael D.

Bentel, Jacqueline M.

Bentley, T. William

Bentley-Hewitt, Kerry L.

Benzeroual, Kenza E.

Berasain, Carmen

Berbel, Pere

Berendsen, Floris F.

Berendzen, Kenneth W.

Berenguer, Juan

Berenguer, Marina

Bergen, Martin von

Berger, Joseph R.

Berger, Terry A.

Berjano-Zanón, Enrique

Berlien, H.-Peter

Bermejo-Nogales, A.

Bermúdez-Aguirre, D.

Bernard-Gauthier, Vadim

Bernardi, Jamila

Bernards, Matt

Bernerd, Françoise

Bernhardt, Harold S.

Bernier, Louis

Bernini, Roberta

Bernstein, Hans-Gert

Bernt, Kathrin

Berrebi, Patrick

Berret, Jean-françois

Berron, Brad J.

Berruyer, Romain

Berry, John P.

Bertoletti, Antonio

Bertram, Ralph

Berzal-Herranz, Alfredo
Kaminska, Marta

Kaminski, Karol Adam

Kamita, George

Kamitani, S.

Kamo, Masaharu

Kamolz, Lars

Kamouchi, Masahiro

Kamunde, Collins

Kan, Lixin

Kanai, Masashi

Kanaoka, Masahiro

Kanayama, Naohiro

Kanazawa, Ippei

Kanda, Takashi

Kanda, Tatsuo

Kandror, Konstantin

Kaneko, Hideo

Kang, Bum-yong

Kang, Dongchul

Kang, Han Chang

Kang, Hara

Kang, Jong

Kang, Jonghoon

Kang, Kwon-Kyoo

Kang, Kyung Pyo

Kang, Sang Sun

Kang, Taek Won

Kannan, S.

Kanno, Yuichiro

Kanopka, Arvydas

Kantharidis, Phillip

Kanungo, Jyotshna

Kao, Kenneth R.

Kaplan, Johanne M.

Kaplan, Richard

Kappachery, Sajeesh

Kapur, Arvinder

Karaca, Esra

Karakasidis, T.E.

Karalekas, Dimitris

Karapanagiotis, Ionnis

Kararigas, Georgios

Karasin'ski, Janusz

Kärenlampi, Sirpa

Karginov, Fedor

Karkhanis, Vrajesh

Karlic, Heidrun

Karni, Rotem

Karp, Effrey M.

Karpukhina, Natalia

Karras, S.N.

Karrich, Julien

Karstedt, S. von
Rochaix, Jean-david

Roche, Daniel

Roche, Philippe

Rochette, Jacques

Rödel, Franz

Rodella, Luigi Fabrizio

Rodino-Klapac, Louise R.

Rodnina, Marina V.

Rodrigo, Ismael

Rodrigues, Clara F.

Rodríguez, Esperanza

Rodríguez, Manuel José

Rodriguez-Frias, F.

Rodríguez-Llorente, I.D.

Rogister, Bernard

Roh, Changhyun

Roh, Hajung

Roh, Sangho

Rohs, Remo

Roidl, Andreas

Rolli-Derkinderen, M.

Roman, Sabiniano

Romano, Giovanna

Romero, Alejandro

Romero, Miguel

Rompel, Annette

Rondanelli, Mariangela

Ropka-Molik, K.

Roppolo, Ignazio

Rosa, Joaquín

Rose, Aaron H.

Rose, Christopher F.

Rose-John, Stefan

Rosell, Rafael

Rosellini, Daniele

Rosenberg, Helene

Rosenmann, Hanna

Rosenzweig, Derek

Ross, Ashley E.

Ross, M.K.

Rossi, Luisa

Rostaing, Lionel

Rota, Rossella

Rotenberg, Susan A.

Rousseau, G.

Routray, Winny

Rowe, Christopher L.

Rowland, Teisha

Roy, Deodutta

Roy, Kunal

Roy, Rosa

Roy, Somak

Royo, Eva 
Besenius, Pol

Betancor, M.B.

Betancur, Catalina

Betarbet, Ranjita

Bethanis, Kostas

Bethke, Gerit

Betoret, Ester

Beversdorf, David

Beyth, Nurit

Bhadauria, Vijai

Bhamidimarri, K.R.

Bhatia, Neehar

Bhatnagar, Vibha

Bhutani, Nidhi

Bhuvaneswari, R.

$\mathrm{Bi}$, Enguang

Bialecka, Monika

Biase, Dario de

Bidarra, Silvia

Bielen, A.A.M.

Bierenstiel, Matthias

Biga, Peggy R.

Bigall, Nadja C.

Bikiaris, Dimitrios

Billette, Christophe

Biocca, Silvia

Birner-Gruenberger, Ruth

Biron, David G.

Bisecco, Alvino

Bishop, Anthony C.

Biswas, Pradip K

Bjarnsholt, Thomas

Bjornstad, Petter

Blais, Anne

Blakley, Brian

Blanco, Rosa M.

Blaner, William

Blankesteijn, W. Matthijs

Błaszczyk, Lidia

Blázquez-Castro, Alfonso

Bleackley, Mark R.

Blennerhassett, Michael

Blomberg, Anders

Blouin, Christian

Blyme, Adam

Bobryshev, Yuri

Boccardo, Francesco

Bochicchio, Brigida

Bochman, Matthew L.

Bock, C.-T.

Bock, Dan G.

Boehm, Volker

Bogel-Łukasil, Rafał
Karten, Barbara

Karumanchi, Ananth

Kasanen, Risto

Kashiwada, Shosaku

Kasper, Maria

Kassiri, Zamaneh

Katagiri, Wataru

Kataoka, Hiromi

Kataoka, Masatoshi

Kataoka, Naoyuki

Kataoka, Yosky

Katayama, Shigeru

Katiyar, Santosh K.

Kato, Ken

Kato, Koichi

Kato, Masaya

Katsila, Theodora

Katsoris, Panagiotis

Katsuma, Susumu

Kattner, Lars

Kauppinen, Anu

Kawabe, Jun-ichi

Kawaguchi, H.

Kawahara, Atsuo

Kawahara, Ei

Kawai, Toshiyuki

Kawai, Vivian K.

Kawakami, Susumu

Kawashima, Hideki

Kay, Euan

Kazeto, Yukinori

Kazu, Kotani

Keedwell, Edward

Kegeles, Lawrence

Keiderling, Timothy A.

Kellenberger, Stephan

Keller, Andreas

Kellner, Franziska

Kelly, Brian N.

Kelly, Christopher V.

Kemp, Stephan

Kennedy, John G.

Kennedy, Patrick

Kenyon, William J.

Kerchev, Pavel

Kerkis, Irina

Kermanshahi-pour, A.

Kern, Matthias

Kersten, Sander

Keshavarz, Mohsen K.

Kessler, Sonja

Keusgen, Michael

Kevin, Gaston
Różalska, Barbara

Rozhon, Wilfried

Ruan, H.D.

Rubbi, Carlos

Rubinow, Katya B.

Rubio, Vicente

Rudat, Jens

Rudkowska, Iwona

Rugarli, Elena I.

Ruigrok, Amber N.V.

Ruiu, Luca

Ruiz, Héctor A.

Ruiz, T. Pena

Rullier, Anne

Rulten, Stuart L.

Rund, Deborah

Ruonala, Mika

Russell, Jacob A.

Russell, Pamela J.

Russo, Aniello

Russo, Nino

Ruthstein, Sharon

Ruvo, M.

Ryan, David K.

Ryan, Michael J.

Rychly, Joachim

Rylott, Elizabeth L.

Sabaté, José

Sabatier, Laure

Sabbaj, Steffanie

Sabbatini, Simona

Sabbatino, Francesco

Sabotič, Jerica

Sacchetti, Lucia

Sadee, Wolfgang

Sadhasivam, S.

Sadik, Riadh

Sadiq, Muhammad W.

Sadoghi, Patrick

Saeki, Kazuhiko

Saenen, Eline

Safa, Ahmad

Saferding, Victoria

Saggioro, Danielle

Sagi, Satyanarayanaraju

Sagredo, Onintza

Sahoo, Sushmita

Sahrawy, Mariam

Said, Ahmed M.

Saisho, Y

Saito, Kuniaki

Saito, Yoshiro

Saitoh, Kenji 
Boger, Heather A.

Boiani, Michele

Boisclair, Yves

Boles, Blaise R.

Bolmontemail, Tristan

Bolotin-Fukuhara, Mme M.

Bols, N.C.

Bondy, Stephen

Bonetti, Bruno

Bongiovanni, Laura

Bonikowski, Radosław

Bonin, Serena

Bonner, William

Boo, Yong Chool

Booij, Linda

Boonstra, André

Borgese, Nica

Borgiani, Paola

Borhan, Mohammed H.

Borich, Michael R.

Bormann, Jörg

Borrelli, Joseph, Jr.

Bortolozzi Biassoni, A.

Boscá, Lisardo

Boscaiu, Monica

Bose, Jayakumar

Boshoff, Helena

Bosland, Paul

Bosnjak, Berislav

Both, Sanne

Böttcher, Yvonne

Bouaïcha, Noureddine

Bouaziz, Serge

Boucheix, Claude

Boughton, Berin

Boulding, E.G.

Bourgault, Steve

Bourgeois, Cyril

Bourgeois, Dominique

Boutin, Jean

Bowden, Scott

Bowman, Teresa V.

Bowness, Paul

Boyd, Chris

Boyer, Anne E.

Boyle, Joseph J.

Brader, Günter

Braga, Valdir A.

Braig, Simone

Brakmann, Susanne

Brandner, Sebastian

Braslavsky, Silvia E.

Braun, Ralf J.
Keyghobadi, Nusha

Khan, Salma

Khanna, Nidhi

Khanna, Savita

Khiari, Zied

Khorram, O.

Khriachtchev, Leonid

Kiaris, Hippokratis

Kiddle, Guy

Kiemnec-Tyburczy, K.M.

Kietzmann, Thomas

Kikuchi, E.

Kikuchi, Haruhisa

Kikuchi-Ueda, Takane

Kim, Beom Joon

Kim, Chang Ju

Kim, Chun Ho

Kim, Dong Joon

Kim, Dong-Pyo

Kim, Eun-Cheol

Kim, Hoon

Kim, Hugh I.

Kim, Hye Kyong

Kim, Hyeung-Rak

Kim, Il-man

Kim, Jae Young

Kim, Jae-jin

Kim, Jae-Sung

Kim, Jin Moon

Kim, Jin-Wook

Kim, Joong Sun

Kim, Julian

Kim, Jung Bae

Kim, Kiyoung

Kim, Kyoung Soo

Kim, Kyung Soo

Kim, Kyung-su

Kim, Moon Ki

Kim, Na-hyung

Kim, Okjoon

Kim, S.J.

Kim, Sang-We

Kim, Soonhag

Kim, Sunghoon

Kim, Sung-Hoon

Kim, Sungtae

Kim, Sungwhan

Kim, Woo Kyun

Kim, Wun-Jae

Kim, Yong Soo

Kim, Yonghee

Kim, Youngsoo

Kim, Yun-bae
Saitoh, Noriko

Sakakibara, Iori

Sakamoto, Kei

Sakamoto, Yuichi

Sakhaee, Khashayar

Saku, Takashi

Sakuma, Kunihiro

Sakuragi, Yumiko

Sakurai, Minoru

Salbitani, Giovanna

Saleem, Mohammad

Saleem, Muhammad

Saleh, Fatima A.

Salehi-Reyhani, Ali

Saliba, Anthony J.

Saliu, Francesco

Sallé, Guillaume

Salo-Ahen, Outi M.H.

Salsano, Ettore

Salvadó, Miguel A.

Salvatore, Francesco

Salvi, Anna M.

Samaha, Anne-Noël

Samarasinghe, Sandhya

Samavati, Lobelia

Samia, Anna

Sammons, Robert D.

Sampath, Prabha

Sams, Thomas

Samuel, Marcus

Samuels, Mark E.

Sanak, Marek

Sánchez Alcázar, José

Sanchez, Diego H.

Sánchez, María-teresa

Sanchez-Amat, Antonio

Sánchez-Fortún, $\mathrm{S}$.

Sánchez-Pernaute, Olga

Sandjo, Louis P.

Sandkam, Benjamin A.

Sandre, Olivier

Sandri, Giuseppina

Sanejouand, Yves-Henri

Sangadala, Sreedhara

Sangelaji, Bahram

Sanna, Fabio

Sansone, Clementina

Santamaria, Rita

Santander, Javier

Santarsiero, Bernard D.

Santino, Angelo

Santoni, Matteo

Santoro, Nicola 


\author{
Bravaccio, Carmela \\ Brazzelli, Valeria \\ Brazzolotto, Xavier \\ Bredenbeek, P.J. \\ Breitbart, Haim \\ Breitenfeld Granadeiro, L. \\ Breitling, Rainer \\ Brendlé, Jocelyne \\ Brennan-Speranza, Tara C. \\ Brenner, Ingrid \\ Breton, Timothy \\ Brew, B.J. \\ Breydo, Leonid \\ Brijder, Robert \\ Brilot, Fabienne \\ Bringmann, Andreas \\ Brini, Marisa \\ Briza, Peter \\ Brochot, Etienne \\ Brock, Guy \\ Brodmann, Marianne \\ Broggini, Massimo \\ Broom, Neil D. \\ Brosh, Robert M. \\ Brouard, Sophie \\ Brown, Chris M. \\ Brown, James \\ Brown, Mark A. \\ Brown, Robert \\ Brown, Susan \\ Bruce, Neil C. \\ Bruckmeier, Rupert \\ Brudzynski, Katrina \\ Bruining, Nico \\ Brumm, Phillip J. \\ Brunaud, Véronique \\ Brunner, Michael \\ Bruno, John \\ Bruserud, Øyvind \\ Bryson-Richardson, R. \\ Bubici, Giovanni \\ Buckley, M. \\ Buckner, Jane H. \\ Bucolo, Claudio \\ Budunova, Irina \\ Buechler, Christa \\ Bugg, Timothy D.H. \\ Bulla, Lee A., Jr. \\ Bullon, Pedro \\ Bumgardner, Joel D. \\ Buonaguro, Elisabetta F. \\ Buono, P. \\ Buonocore, Francesco
}

Kiminobu, Sugaya

Kimira, Yoshifumi

Kimura, Hideto

King, Aileen

King, Paul W.

King-Smith, P. Ewen

Kinugawa, Shintaro

Kipp, Anna P.

Kira, Jun-ichi

Kirby, Ralph

Kirchhoff, Frank

Kirschning, Carsten J.

Kirsebom, Leif

Kiss, István Z.

Kiss, Margaret M.

Kiss, Robert

Kisselev, Alexei

Kitade, Yukio

Kitajima, Shigetaka

Kitlinska, Joanna

Kittle, David S.

Kizaka-Kondoh, Shinae

Klaren, Rachel

Klein, Kerstin

Kliem, Heike

Klintsova, Anna

Klitgaard, Janne

Kloiber, Stefan

Klose, Johannes

Kluza, Jérome

Knapp, Stefan

Knauer, Shirley K.

Knetsch, Menno L.W.

Knölker, Hans-Joachim

Knoshaug, Eric

Knott, Rachel

Ko, Jane L.

Kobayashi, Hiroshi

Kobayashi, Kazuo

Kobayashi, Yoshiro

Kobeissy, Firas H.

Kobel, Martin

Köcher, Thomas

Kocmarek, Andrea L.

Koda, M.

Kodama, Daisuke

Koehbach, Johannes

Koes, David Ryan

Köfeler, Harald

Koff, Andrew

Kofuji, Kyouko

Koga, Fumitaka

Kogut, Michael H.
Santos, Catarina

Santos, Isabel

Santos, Teresa Almeida

Santulli, Gaetano

Sanz, Luis

Saravanan, Palaniappan

Sardon, Haritz

Sarkar, Sibaji

Sasagawa, Toshiyuki

Sasaki, Hikaru

Sathishkumar, K.

Sato, Aaron K.

Sato, Kenji

Sato, Naoki

Sato, Shin

Sato, Yutaka

Satoh, Shigeru

Satoh-Nagasawa, Namiko

Saunderson, Sarah

Savard, Christian

Saviano, Michele

Sawada, Kenjiro

Sawalha, Amr H.

Sawers, R. Gary

Sayers, Thomas J.

Scalise, Mariafrancesca

Scarano, Christian

Scatena, Roberto

Schäfer, Michael

Schaller, Hubert

Schatten, Heide

Schaumburg, Frieder

Scheibe, Renate J.

Scheiner, Steve

Schemmer, Peter

Schenke-Layland, Katja

Scherer, Günther

Schernthaner, Johann

Schernthaner, Ruediger E.

Schierle, Gabriele K.

Schiller, Dirk

Schinke, Thorsten

Schirrmacher, Ralf

Schlessinger, Avner

Schluttenhofer, Craig

Schmeiser, Heinz H.

Schmid, J.

Schmiderer, Corinna

Schmidt, Bernd

Schmidt, Marco F.

Schmidt, Marion

Schmidt, Morten

Schmidtchen, Franz P. 
Bürckstümmer, Tilmann

Burg, Kornel

Burger, Gertraud

Burgess, Catherine M.

Burgess, Karl E.V.

Burgoon, Mark P.

Burkhead, Jason L.

Burton, Zachary F.

Busch, Hauke

Busch, Theresa M.

Businaro, Rita

Bustamante, Sonia

Bustin, Stephen A.

Buzina, Walter

Byrt, Caitlin Siobhan

Cabral, Jaydee

Caccamo, Daniela

Cadet, Frédéric

Cai, Hongmei

Cai, Lu

Cai, Weili

Calafiore, Riccardo

Calani, Luca

Calder, Philip

Calder, Phillip

Calderaro, Adriana

Calvano, Cosima D.

Calvisi, Diego F.

Camara, Amadou K.S.

Cameli, Norma

Cammas-Marion, S.

Campbell, Ewan

Campiglia, Pietro

Campo, Giuseppe M.

Camporeale, Annalisa

Campos, Francisco

Camps, Jordi

Cancello, Raffaella

Cancino, Jorge

Canela, Ramon

Canini, Antonella

Cannata-Andía, Jorge

Cannon, Ronald E.

Cano, Amparo

Cano-Delgado, Ana

Cao, Ling

Cao, William

Capasso, Raffaele

Capitanio, Nazzareno

Caraceni, Paolo

Caragea, Doina

Carbonell, Pablo

Carboni, Lucia
Koide, Hikaru

Koizumi, Nozomu

Kojima, Akiko

Kojima, Chie

Kojima, Tetsuya

Kok, Klaas

Kokaze, Akatsuki

Kokura, Satoshi

Kolehmainen, Erkki

Kolialexi, Aggeliki

Kolovou, Genovefa

Kolpashchikov, Dmitry

Komine, Mayumi

Kondo, Eisaku

Kondo, Hidehiro

Kondo, Mitsuru

Kondo, Tatsuya

Konigsberg, William H.

Konishi, Hiroaki

Konstantinou, Ioannis K.

Konstorum, Anna

Kontoyianni, Meropi

Kontro, Inkeri

Koo, In Sun

Koo, Ja Seung

Kook, Koung Hoon

Koole, Leo H.

Kooyman, David L.

Kopczak, Anna

Koppang, Erling

Kopra, Kari

Korach, Kenneth

Korade, Zeljka

Korczyn, Amos D.

Korosi, Aniko

Korsching, Eberhard

Koshino, Hiroyuki

Kosmacz, Monika

Kosova, Klara

Kossida, Sofia

Kostin, Sawa

Kouadio, James Halbin

Koufaki, Maria

Koupenova-Zamor, Milka

Kouretas, Dimitrios

Kourist, Robert

Kourkoumelis, Nikolaos

Kourti, Anna

Kovar, Heinrick

Kowalczyk, Mariusz

Kowalski, Konrad

Kozlowski, Lukasz

Kozyrovska, Natalia
Schmitz-Linneweber, C.

Schmoelzl, Sabine

Schneider, Joel

Schneider, Raphael

Schoenhagen, Paul

Schonthal, Axel

Schoonbeek, Henk-jan

Schrader, Andrea

Schraufstatter, Ingrid U.

Schroeder, Julian I.

Schroeter, Michael

Schroten, Caroline

Schrum, Laura W.

Schu, Peter

Schuh, Alexander

Schüler, Susanne

Schuller, Hildegard M.

Schulze-Tanzil, Gundula

Schunter, Celia

Schuurink, Robert

Schwabe, Robert F.

Schwarz, Wolfgang

Schwarzacher, Trude

Schwarzenbacher, D.

Schweiger, Wolfgang

Schwertfeger, Kaylee

Schweser, Ferdinand

Sciascia, Savino

Scobie, Linda

Scocchi, Marco

Scorziello, Antonella

Scott, Naomi

Scott, Patrick A.

Scovassi, Anna Ivana

Scozzafava, Andrea

Scriven, Paul

Scudiero, Rosaria

Scurrah, Katrina

Seayad, Jayasree

Seca, Ana M.L.

Sedelnikova, Olga A.

Seeger, Christoph

Seeman, Philip

Seenan, Chris

Ségui, Bruno

Seifert, Karlheinz

Seiliez, Iban

Sekiguchi, Miho

Selek, Laurent

Selisko, Barbara

Sempere, Lorenzo

Sen, Aritro

Sen, Nilkantha 
Cardarelli, Francesco

Cardina, John

Carè, Alessandra

Carlberg, Carsten

Carmichael, Gordon G.

Carneiro, Ângela

Carrasco, Pedro

Carrì, Maria Teresa

Carru, Ciriaco

Carter, Wayne G.

Cartland, Siân P.

Carty, David M.

Carugo, Olivero

Carvalho, Ana Luisa

Carvalho, Joana

Carvalho, Luísa

Casaccia, P.

Casarosa, Simona

Caselli, Chiara

Casey, Theresa M.

Casiraghi, Federica

Cassel-Lundhagen, Anna

Castelein, René M.

Castellano, Gloria

Castiglioni, Bianca

Castro, Antonio Jesus

Catalá, Myriam

Catalano, Alessia

Cats, Annemieke

Catts, Vibeke

Cauli, Omar

Cavalla, P.

Cavassini, Matthias

Cavicchi, Kevin

Ceci, Luigi R.

Ceciliani, Fabrizio

Cederbaum, Arthur

Cederberg-Helms, Hans C.

Celetti, A.

Celi, Francesco

Celius, E.G.

Cellerino, Alessandro

Cellini, Francesco

Cervantes, Jorge L.

Cha, Byung-Yoon

Cha, Sangwon

Cha, Tae-Joon

Chae, Sungwook

Chahine, Mohamed

Chai, Lijun

CHAIMBAULT, Patrick

Chalak, Lina F.

Chaleix, Vincent
Kraemer, Fredric B.

Kraemer, Kenneth $\mathrm{H}$.

Kraguljac, Nina Vanessa

Krämer, Oliver H.

Kramer, Phillip

Kratimenos, Panagiotis

Kraus, Virginia

Krauss-Etschmann, S.

Kreader, Carol A.

Krebs, Joachim

Kreienkamp, H.-J.

Krenek, Sascha

Krengel, Ute

Kresanov, Petri

Krishnan, Sitaraman

Kristian, Tibor

Krol, Alain

Kroll, Jens

Kronvall, Erik

Kropski, Jonathan A.

Krueger, R.R.

Kruger, Ruan

Krynetskiy, Evgeny

Krystal, John H.

KS, Chun

Kuan, Yu-Hsiang

Kuang, Shihuan

Kubant, Ruslan

Kubelka, Jan

Kubina, Robert

Kubo, Tomohiko

Kuçi, Selim

Kuehl, Mike

Kuerten, Stefanie

Kuhlmann, Markus

Kuiper, Roland

Kuivaniemi, Helena

Kukolj, George

Kuliawat, Regina

Kulka, Marianna

Kumar, R.

Kumar, Sandeep

Kumar, Ujendra

Kumazawa, Yoshinori

Kume, Toshiaki

Kumi-Diaka, James

Kundakovic, Marija

Kundu, Joydeb Kumar

Kunimasa, Kazuhiro

Kunisada, Takahiro

Kunnimalaiyaan, M.

Kunte, Hagen

Kuntz, Marcel
Sen, Shukdeb

Seneviratne, Chaminda J.

Senthil Kumar, T.

Seoane Fernández, José A.

Seoane, Samuel

Serantes, David

Serata, Masaki

Serpente, Maria

Serra, Serra

Serrano, Irene

Serrano, Jose C.E.

Serrano, María

Seruggia, Davide

Servadei, Franco

Servi, Stefano

Sestili, Sara

Seth, Devanshi

Sethi, Gautam

Severa, Martina

Seybold, Virginia S.

Seyfert, Hans-Martin

Sgambato, Alessandro

Shafi, Tariq

Shah, Aarti

Shaio, Young-ji

Shakibaei, Mehdi

Shalin, Sara

Shamimuzzaman, Md

Shankar, Anil

Shanmugam, M.

Shannahan, Jonathan

Shapiro, Bruce

Sharfstein, Susan T.

Sharifimajd, Babak

Sharma, Naveen

Sharma, Rohit

Shavrukov, Yuri

Shaw, Pang-Chui

She, Qing-Bai

Shehu, Amarda

Shelomi, Matan

Shen, Jian Gang

Shen, Juan

Shen, Leslie

Shen, Szu-Chuan

Shen, Wen

Shen, Yiping

Shen, Yuh-Chiang

Sherman, Louis

Shi, Changbin

Shi, Jiong

Shi, Qiang

Shi, Yang 
Chalkley, Robert

Chalmers, Jeffrey J.

Chamberlain, Jeffrey S.

Chamberlain, Stormy

Chambers, J.E.

Chamcheu, Jean C.

Champion, Antony

Chamulitrat, Walee

Chan, King Ming

Chan, Koon-Ho

Chan, Kun-Ming

Chan, Shiao-Yng

Chan, Sun On

Chan, Wai-Yee

Chandrasekar, Raman

Chang, Cheng

Chang, Chien-hsing

Chang, Chin-Chyuan

Chang, Chiou Ling

Chang, Hao-Teng

Chang, Hsueh-Wei

Chang, Hui-hua

Chang, Hyeun Wook

Chang, Ing-Feng

Chang, John P.

Chang, Jo-Shu

Chang, Long-Sen

Chang, Raymond

Chang, Wei-Chaio

Chang, Yu-Jia

Chapel, Alain

Chapman, Mark A.

Chaput, Gina

Charlton, Clivel

Chase, Andrew

Chater, Caspar

Chatonnet, Arnaud

Chatterjee, Jhinuk

Chaudhry, Parvesh

Chavez, Ferman

Chen, Bin

Chen, Chih-cheng

Chen, Chongyi

Chen, Chung-Ming

Chen, Chunjung

Chen, Dong

Chen, Eleanor

Chen, Guang-Chao

Chen, Houyang

Chen, Hsien-Jung

Chen, Jiann-Chu

Chen, Jing-Hsien

Chen, Jin-Gui
Kunz, Wolfram

Kunze, Gotthard

Kuo, Cheng-Chin

Kuo, Hann-Chorng

Kuo, Tzong-Fu

Kuramochi, Kouji

Kurasaki, Masaaki

Kurata, Y.

Kurdowska, Anna K.

Kurgan, Lukasz A.

Kurihara, Daisuke

Kurita, Hirofumi

Kurose, Hitoshi

Kurschus, Florian C.

Kurumizaka, Hitoshi

Kusakabe, Makoto

Kusakabe, Takahiro

Kuschal, Christiane

Kushibiki, Toshihiro

Kusumoto, Ken-Ichi

Kutschera, U.

Kuuskeri, Jaana

Kuwata, Keith T.

Kwa, Faith A.A.

Kwan, Joseph S.K.

Kwok, Seung-Ki

Kwon, Il-Keun

Kwon, Oh-Seung

Kwon, Tae Gyun

Kwon, Young-Wan

Kyong, Jin Burm

Kyrø, Cecilie

Kyttaris, Vasileios C.

Kyzas, George Z.

La Rocca, Renato V.

Laederach, Alain

Lagace, Thomas A.

Lage, Cerenius

Lagerstedt, Jens O.

Lai, Michael M.C.

Laidlaw, Clinton T.

Lakin-Thomas, Patricia L.

Lakka, S.

Lala, Ranjna Madan

Lalman, Jerald

Lam, Jenny K.W.

Lam, Minh

LaMarca, Babette

Lambert, Elisabeth

Lambertsen, Kate Lykke

Landar, Aimee

Landis-Piwowar, Kristin

Lang, Andrew
Shigeru, Sato

Shih, Chun-Che

Shillcutt, Samuel

Shim, Yhong-hee

Shimada, Tsutomu

Shimizu, Takahiko

Shimoda, Kazuya

Shimono, Yoshiko

Shimosawa, Tatsuo

Shin, Ki-Hyuk

Shin, Shyi-Jang

Shin, Ueon-sang

Shinohara, Russell T.

Shintani, Yasushi

Shintani, Yoshinori

Shiomitsu, Keijiro

Shiota, Masaki

Shiozawa, Mikio

Shiraha, Hidenori

Shiraishi, Takehiko

Shirakami, Yohei

Shirure, Venktesh

Shivapurkar, Narayan

Shmizu, Masahito

Shoda, Makoto

Shoji, Sunao

Shoji, Tsubasa

Shomura, Yasuhito

Shoubridge, Cheryl

Shoyama, Yukihiro

Shrestha, Lok Kumar

Shrestha, Pravin Malla

Shui, Irene M.

Shynlova, Oksana

Shyu, Ann-Bin

Siah, Ahmed

Sicca, Federico

Sicher, Richard C.

Siciliano, Carlo

Siculella, Luisa

Sidorenko, Alexander

Sieber, Jonas

Siegmund, Sören V.

Sienkiewicz, Andrzej

Signorini, Cinzia

Sigusch, Bernd W.

Sikva, Artur M.S.

Sill, Heinz

Silljé, Herman H.W.

Siltanen, Antti

Silva, Gabriela

Silva, J.C.

Silva, Luís R. 
Chen, Ji-Yih

Chen, Juan

Chen, Lin-Chi

Chen, Min

Chen, Peng-Wen

Chen, Po-Yuan

Chen, Qi

Chen, Qi-Yin

Chen, Ruei-ming

Chen, Szu-Fu

Chen, Tzong-Yueh

Chen, Wen-Cheng

Chen, Xi

Chen, Ya-Wen

Chen, Yen-Chou

Chen, Ying-Pin

Chen, Yu Wai

Chen, Yung-Hsiang

Chen, Zhe-Sheng

Chen, Zhonghua

Chen., Cheng-Nan

Cheng, Fang-Yu

Cheng, Jianlin

Cheng, Jing-Jy

Cheng, Juei-tang

Cheng, Kai

Cheng, Xingguo

Cheng, Xuanhong

Cheng, Yang-tse

Cheng, Yong

Cheng, Zhiliang

Cheon, Choong-ill

Cheon, Yong-Pil

Cheong, Yong Hwa

Chermette, Henry

Chern, Ming-Kai

Chevalier, Xavier

Chiang, Han-Sun

Chiang, Tai-an

Chiang, Wen-Dee

Chiappini, Ciro

Chiappori, Alberto A.

Chiechio, Santina

Chien, Chih-Ching

Chiesa, Roberto

Chimenti, Sergio

Chio, Chung-Ching

Chio, In-Hong

Chiorino, Giovanna

Chiou, Hui-ling

Chiou, Wen-fei

Chirumbolo, Salvatore

Chiu, Bernard
Lang, Siegmund

Lange, Kathrin M.

Langridge, Peter

Lanham, S.A.

Lanni, Antonia

LaPatra, Scott

Lapierre, Pascal

Laplante, Mathieu

Lapres, John J.

Larizza, Lidia

Larkin, Philip J.

Larkum, Anthony

Larrainzar, Estibaliz

Larsen, Vanessa Garcia

Larson, Nicholas B.

LaRue, Amanda C.

Lash, Lawrence

Lasserre, Bruno

Latosińska, Jolanta $\mathrm{N}$.

Latour, Xavier

Latte, Gianmarco

Lattuada, Marco

Lau-Cam, Cesar A.

Laughlin, Maren R.

Laulagnier, Karine

Lauro, M.R.

Lausen, Bertold

Lavandera, Ivan

Lavelli, Vera

LaVoie, Holly A.

Lawrence, Clare L.

Lawrence, Susan D.

Lay, Jackson O.

Layden, Michael

Layden, Michael J.

Layh-Schmitt, Gerlinde

Lazzeri, Chiara

Le Borne, Sylvie

Le Prell, Colleen

Le Questel, Jean-yves

Le, Lu Q.

Lebleu, B.

Leboeuf, Celine

Lechner-Scott, Jeannette

Leclercq, Julie

Ledesma, Maria Dolores

Ledoux, Mark

Leducq, Jean-Baptiste

Lee, ChangWoo

Lee, Cheng-Kuo

Lee, Chi

Lee, Chia-hung

Lee, Chong Jae
Silva, M. Luísa S.

Silva, Sn

Silveira-Moriyama, Laura

Silvestri, Elena

Simal Gándara, Jesús

Simirgiotis, Mario J.

Simitzis, Panayiotis

Simões, M.C.F.

Simon, Richard

Simon, Ronald

Simonović, Miljan

Simonsen, Henrik Toft

Singamneni, Sarat

Singaravelu, G.

Singer, Cherie A.

Singh, Ashok K.

Singh, Chandra K.

Singh, Jagdish

Singh, Kameshwar P.

Singh, Karan

Singh, Kashmir

Singh, Seema

Singh, Udai P.

Sinha, Krishna M.

Sinnott, Michael L.

Sintim, Herman O.

Sirimulla, Suman

Sirota, Fernanda L.

Sirtori, Federico Riccardi

Siskind, Leah J.

Sissler, Marie

Sissung, Tristan M.

Siu, Parco M.

Sjogren, Klara

Skaf, Dorothy W.

Skinner, Jonathan

Skold, Helen Nilsson

Skøt, Leif

Skripuletz, Thomas

Skvortsova, Ira

Slack, Frank

Sleebs, Brad E.

Sleiman, Patrick M.A.

Slimestad, Rune

Slominski, Andrzej

Slovin, Susan

Smail , Hadj-Rabia

Smart, Neil

Smielewski, Peter

Smiesko, Martin

Smirnovas, Vytautas

Smith, Alicia K.

Smith, Andrew 
Chiu, Tsai-Hsin

Chiurchiù, Valerio

Cho, Dong-Woo

Cho, Mi-La

Cho, Yong-Gu

Chobot, Vladimir

Choi, Byung Tae

Choi, Byung-Kwon

Choi, Donghoon

Choi, Eung $\mathrm{H}$.

Choi, In-Geol

Choi, Inho

Choi, In-young

Choi, Seok Ki

Choi, Yoo Seong

Choi, Yung Hyun

Chomilier, Jacques

Chong, Parkson Lee-gau

Chopp, Michael

Chorianopoulos, Nikos G.

Chou, C. James

Chou, Kuo

Chou, Wing-ming

Choudhury, Swarup Roy

Chougule, Mahavir B.

Chow, Cheuk Fai Stephen

Chow, Wang-Ngai

Chowdhury, Golam M.I.

Chowdhury, Kamal

Christ, Bruno

Christensen, Eidi

Christiansen, Christian F.

Christofidou-Solomidou, M.

Christov, Christo Z.

Chrzanowski, Łukasz

Chu, Xiangping

Chuang, Wan-Long

Chueh, Pin Ju

Chun, Heung Jae

Chun, Jerold

Chung, Euiryong

Chung, Wen-Hung

Chung, Ying-Chien

Chwalibog, André

Chyau, Charng-Cherng

Ciaccio, Marcello

Ciappellano, Salvatore

Ciardelli, Gianluca

Cichero, Elena

Cicuta, Pietro

Cillo, Clemente

Cindrova-Davies, Tereza

Ciofani, Gianni
Lee, Choong Hwan

Lee, Dae Ho

Lee, Dong Gun

Lee, Duu-Jong

Lee, Eun Kyung

Lee, Eun Woo

Lee, EunAh

Lee, Gabsang

Lee, Gsm

Lee, Hsin-Chen

Lee, Huei

Lee, Hwa Jin

Lee, Hye Suk

Lee, Jai-Wei

Lee, Jeong I.K.

Lee, Juliana Tsz Yan

Lee, Jung C.

Lee, Jungwha

Lee, Justin

Lee, Kin Wah Terence

Lee, Kisay

Lee, Kwang Ho

Lee, Kwan-Sung

Lee, Kyu Wan

Lee, Leo T.O.

Lee, Lin-Wen

Lee, Min Won

Lee, Peng

Lee, Rong-Ho

Lee, Sang Kook

Lee, Sang Yeol

Lee, Sang-han

Lee, Sanghyeob

Lee, Sang-Won

Lee, Seong-Ryong

Lee, Shyi-Long

Lee, Sung Chul

Lee, Taeseung

Lee, Tzong-shyuan

Lee, Vincent Ws

Lee, Wing-kee

Lee, Won Sup

Lee, Won-Ha

Lee, Wooin

Lee, Wooyoung

Lee, Yoon Kwang

Lee, Young

Lee, Yu-Hsiang

Leffler, Jonatan

Lefterov, Iliya

Legendre, Laurent

Legouin-Gargadennec, B.

Lehmann, Alan R.
Smith, Anja Van Brabant

Smith, Clyde A.

Smith, Darrin

Smith, Elizabeth R.

Smith, Geoffrey R.

Smith, Jennifer A.

Smith, Judith A.

Smolock, Christopher J.

Snyder-Cappione, J.E.

Sobarzo-Sánchez, E.

Sobczak-Thépot, Joëlle

Socol, Yehoshua

Sodi, Anna Mensuali

Sodupe, Mariona

Sofo, Adriano

Soga, Yoshimitsu

Sogorb, Miguel A.

Soilu-Hänninen, M.H.

Sokilde, Rolf

Sokolova, Viktoriya

Soleimani, Majid

Soll, Dieter

Sommer, Wolfgang $\mathrm{H}$.

Somogyi, Arpad

Son, Mi-Won

Sone, Hideko

Song, Guoqing

Song, Gwan Gyu

Song, Hae-Ryong

Song, Jianxun

Song, Jonathan

Sonsalla, Patricia K.

Soon, Yu Yang

Soreq, Hermona

Soriani, Olivier

Soriano, Elena

Soric, Audrey

Sotriffer, Christoph

Souders, Colby A.

Souza-Smith, Flavia M.

Sovak, Guy

Spagnuolo, Antonietta

Spagnuolo, Carmela

Spalletta, Gianfranco

Spandou, Evangelia

Spano, Giuseppe

Sparks, Paul B.

Sparrow, Janet R.

Sparvoli, Francesca

Spelman, Lynda

Spengler, Ulrich

Spiess, Christoph

Spinazzola, Antonella 
Cirillo, Giuseppe

Cirone, $\mathrm{M}$.

Citron, Bruce A.

Clark, Shannon

Clarke, Anthony

Claudia, Grothe, P.

Claudia, Volpi

Claus, Harald

Clavel, Marie-Annick

Clawson, Gary A.

Clem, Brian F.

Clément, Gilles

Clementi, Nicola

Clendenen, Tess V.

Cleveland, Beth M.

Cloos, Jacqueline

Coates, Christopher J.

Cobb, Joanna

Cocetta, Giacomo

Cockerill, Gillian

Codacci-Pisanelli, G.

Coelho, José A.

Cognasse, Fabrice

Cohen, Adam

Cohen, Yoram

Cohick, Wendie

Cohney, Solomon

Colbert, Robert A.

Cole, Steve

Coleman, David

Coleman, William B.

Coles, Alasdair J.

Coll, Josep

Colle, Isabelle

Colliec-Jouault, Sylvia

Collins, Helen M.

Colombié, Sophie

Colombo, C.

Coluccia, E.

Concas, Alessandro

Concordet, Jean-Paul

Conlon, Michael A.

Constanti, Magda

Conte, Pierfranco

Conti, Ario

Contini, Alessandro

Cook-Mills, Joan M.

Cooper, Arthur

Cooper, William T.

Coppin, Hélène

Coppola, David M.

Coppola, Nicola

Corcoran, Brendan M.
Lehmann, Christian

Lehto, Taavi

Lei, Xia

Leigh, Spencer A.

Leitão, Ana

Leitz, Thomas

Lembo, Serena

LEN, Christophe

Lenaz, Giorgio

Lennartsson, Patrik R.

Lenon, George

Leonard, Nola

Leone, Alessandro

Leong, David Tai

Leopold, Jane A.

Lephart, Edwin D.

Leppla, Stephen H.

Lerman, Lilach

Lessard, Christopher

Lessman, Charles

Leucht, S.

Leung, Chung-Hang

Leung, David

Leung, Ping-Chung

Leung, Yuet-Kin

Leuven, Fred Van

Levine, Andrew J.

Levraud, Jean-pierre

Lewandowski, Bartosz

Lewin, $\mathrm{Al}$

Lewinson, Oded

Lewis, Michael

Lewis, Ramsey

Lewkowich, Ian P.

Ley, Alexandra C.

Ley, Steve

Leygue, Etienne

Leyrat, Cedric

Lezot, Frédéric

Li, Bing

Li, Chi

Li, Defang

Li, Guosheng

Li, Hsing-Hui

$\mathrm{Li}$, Jianming

Li, Jinsong

Li, Kuo-Bin

$\mathrm{Li}$, Lei

$\mathrm{Li}, \mathrm{Li}$

Li, Ling

Li, Mei-Ling

Li, Ming

Li, Qinghong
Spitzner, Melanie

Spizzo, Gilbert

Spoelder, Jan

Sredni, Simone T.

Srinivas, Keerthi

Srivatsan, Malathi

Srivenugopal, Kalkunte

Staege, Martin S.

Staff, Anne Cathrine

Stamatatos, Theocharis C.

Stamelos, Vasileios

Stammler, Gerd

Stanley, Joanna

Stansfield, Kirstie

Stanta, Giorgio

Stanton, Robert C.

Starostik, Petr

Stassi, Giorgio

Staudacher, Alexander H.

Stead, Lucy F.

Steardo, Luca

Stec, David E.

Steen, Andrew D.

Steenwijk, Martijn D.

Stefanidou, Maria E.

Stefano, Cassanelli

Stefano, Cesco

Steffens, Bianka

Stehouwer, Peter Paul

Steigedal, Tonje S.

Steinritz, Dirk

Stepensky, David

Steuer, Andrea E.

Stevanato, Piergiorgio

Stevens, Stanley

Stevenson, Graeme

Stiborova, Marie

Stich, Stefan

Stiegelbauer, Verena

Stocco, Gabriele

Stock, Peggy

Stockmann, Regine

Stoddard, Barry L.

Stoecklin, Georg

Stone, Trevor William

Stope, Matthias B.

Stournaras, Christos

Strehmel, Nadine

Stricker, Sigmar

Strom, Jakob O.

$\mathrm{Su}$, Chun-Li

Su, Chunming

Su, Dong-Ming 
Cordero, Mario

Cordero, Paul

Corey, Seth J.

Corley, M.M.

Corporeau, Charlotte

Correia, Sónia C.

Cortes, Andres J.

Cortez, M.M.

Cos, Paul

Cosentino, Ugo

Cosgrove, Daniel

Cosma, Pinalysa

Cossigny, Davina

Costa, Valerio

Costantini, David

Costa-Rodrigues, João

Costoya, Jose A.

Côté, Jean-François

Cotella, Dott Diego

Coto, Mairene

Couffin, Anne-Claude

Couttet, Philippe

Couture, Réjean

Cova, Lucyna

Covès, Jacques

Cragg, Peter J.

Crago, Jordan

Craig, Paul M.

Crescioli, Clara

Creusot, Nicolas

Crews, Colin

Crisanti, Andrea

Critchley, Julia

Croce, Fausto

Cronin, Mark

Crossman, David J.

Crosthwaite, Susan K.

Cruchaga, Carlos

Csuk, René

Cueto, Mercedes

Cui, Jun

Cullis, Christopher

Culman, Juraj

Curci, John A.

Curiel, Laura

Curley, Gerard F.

Czosnyka, Zofia

Da Silva, Douglas Ganini

Da Silva, M.L.Alves

Da Silva, Mateus Webba

Dacher, Matthieu

Daglia, Maria

Dai, Tianhong
Li, Quan-Zhen

Li, Ronald

Li, Siheng

Li, Tiangang

Li, Wen-hong

Li, Xiaohua

Li, Xiaohui

Li, Xiaoli

Li, Xudong Joshua

Li, Xuewei

Li, Yefu

Li, Yi

Li, Yong

Li, Yongfang

Li, Yu-you

Lian, Christine G.

Lian, Jane B.

Liang, Xue-Hai

Lianidou, Evi S.

Liao, Jiunn-wan

Liao, Jyh-Fei

Liao, Ping

Libert, Sergiy

Liebisch, Gerhard

Liehn, Elisa A.

Liekens, Sandra

Lienkamp, Karen

Liermann, Johannes C.

Lim, Dongwook

Lim, Jong-Seok

Lim, Keng Gat

Lim, Kyung-Min

Lim, Siew Pheng

Lim, Sung-Jig

Lim, Young-Cheol

Lima, Renata

Limam, Ferid

Lin, Cheng-Wen

Lin, Chi-Chen

Lin, Chih-Chien

Lin, Chiou-feng

Lin, Glenn

Lin, Ho

Lin, Hung Wen

Lin, Hungdu

Lin, Hung-Yin

Lin, Kui

Lin, Kwang-Huei

Lin, Li

Lin, Ping-Ting

Lin, Qinsong

Lin, Qiong

Lin, Qisheng
$\mathrm{Su}, \mathrm{Hua}$

$\mathrm{Su}$, Jui-Hsin

$\mathrm{Su}$, Tsung-Ping

Su, Tung-Hung

$\mathrm{Su}$, Wen-Da

$\mathrm{Su}, \mathrm{Yeu}$

Suárez, Juan

Suenaga, Hikaru

Suetsugu, Shiro

Sugawara, Akira

Sugawara-Narutaki, Ayae

Sugimoto, Yoshiaki

Sugimura, Haruhiko

Sugita, Noriko

Sugiyama, Hiroshi

Suh, Dae-yeon

Suh, Hyung Joo

Suh, Pann-Ghill

Suhling, Klaus

Sukumaran, Anil

Sullivan, Shannon D.

Suman, Devi S.

Sumby, Krista

Sumi, Shoichiro

Sumich, Alex

Sumner, Katie

Sun, Dandan

Sun, Genlou

Sun, Raymond Wai-Yin

Sun, Weimin

Sun, Ying

Sun, Zhonghua

Sundin, George

Sung, Ping-Jyun

Sung, Sibum

Sunseri, Francesco

Supiot, Stéphane

Supowit, Scott C.

Supuran, Claudiu T.

Surai, Peter

Sureshkumar, Kalathil K.

Surette, Marc E.

Surguchov, Andrei

Suter, Marc R.

Suter, Melissa A

Sutherland, John B.

Suyama, Mikita

Suzuki, Hitoshi

Suzuki, Kaoru

Suzuki, Masataka

Suzuki, Noboru

Svensson, Katrin J.

Svircev, Antonet M. 
Dai, Wenhao

Dai, Zhaohua

Dai, Zong

Daiber, Andreas

Dakhama, Azzeddine

Dalbies, Rozenn

D'all aqua, Stefano

Dalmeijer, Gerdien

Dalrymple, Brian P.

Damalas, Christos

Danby, F. William

Dane, Fenny

Danesi, Francesca

Danser, Jan

Darabi, Hatef

Darder, Margarita

Dare, Andrew

Darvin, Maxim

Das, Anindita

Das, Chittaranjan

Das, Raj

Das, Samarjit

Das, Subha R.

Das, Suman

Dasmahapatra, Asok

Dave, Kunjan R.

D'Aversa, Teresa G.

David, Jonathan

David-Cordonnier, M.-H.

Davide, Degli Esposti

Davidson, Sean M.

Davidson, William S.

Davies, Thomas G.E.

Davis, Gregory D.

Davis, Michael E.

Davis, Mindy I.

Day, Andrew

Day, Regina

Dayal, Sanjana

Dayan, Dan

De Berardis, Domenico

De Bernonville, T.D.

De Braud, F.

De Bruin, Christiaan

De Carolis, Caterina

De Feo, Vincenzo

De Geest, Bart

De la Campa, Adela G.

De la Escosura, Andrés

De la Fuente, Helena

De la Fuente, Jesus M.

De la Fuente, Leonardo

De la Mata, Javier
Lin, Shuhai

Lin, Shu-ping

Lin, Shyh-Hsiang

Lin, Wan-wan

Lin, Yongjun

Lin, Yun-lian

Lindeman, Jan

Linder, Stig

Lindholm, Dan

Lindholm-Perry, A.K.

Lindner, Daniel

Lindqvist, Per

Lindsay, Christopher D.

Linert, Wolfgang

Ling, Maurice $\mathrm{Ht}$

Ling, Xuefeng B.

Ling, Yong-Chien

Lionetti, Lillà

Liou, Ying-Ming

Lipinski, P.

Lipkowski, Jacek

Lipton, Jeffrey I.

Litescu, Simona C.

Litinas, Konstantinos E.

Liu, Chen-Hua

Liu, Delong

Liu, Feng

Liu, Fwu-Hsing

Liu, Hui-Hsuan

Liu, Jian

Liu, Jin

Liu, Jingwen

Liu, Leyuan

Liu, Liang

Liu, Man

Liu, Ruiqiang

Liu, Shujing

Liu, Wang-Ta

Liu, Wei

Liu, Xiaoyan

Liu, Yanqun

Liu, Ying

Liyana-Arachchi, T.

Lizard, Gérard

Llorente, Briardo

Lo, Huang-Mu

Lo, Mindy S.

Lobodin, Vladislav V.

Locatelli, Marcello

Lockridge, Oksana

Lodola, Alessio

Loh, Amos Hong Pheng

Lomakin, Ivan B.
Swahari, Vijay

Swain, Martin

Swales, Catherine

Swaminathan, Subramani

Swarts, Benjamin M.

Sweet, Kendra

Sweetman, Dylan

Swevers, Luc

Swiecicki, Jean-Marie

Swindle-Reilly, Katelyn E.

Sykiotis, Gerasimos P.

Szabò, Ildikò

Szarvas, T.

Szczubiałka, Krzysztof

Sze, Stephen Cho Wing

Taanman, Jan-Willem

Tabata, Yasuhiko

Tada, Hayato

Tada, Yuichiro

Taddei, Maria Letizia

Tafi, Andrea

Tågerud, Sven

Taglietti, Angelo

Tago, Megumi

Taguchi, Yoshihiro

Taheri, Arash

Tahtamouni, Lubna H.

Tain, You-Lin

Taipaleenmäki, Hanna

Taira, Junsei

Tajbakhsh, J.

Tajima, Kenji

Tajiri, Naoki

Takagi, Kiyoshi

Takahara, Mitsuyoshi

Takahashi, Kazuhiro

Takahashi, Wataru

Takahisa, Kanekiyo

Takaki, Akinobu

Takami, Seiichi

Takami, Taro

Takaoka, Akinori

Takashi, Yagi

Takeda, Youhei

Takehana, Yusuke

Takekoshi, Susumu

Takemori, Hiroshi

Takenaka, Yasuhiro

Takeshita, Fumitaka

Takeshita, Haruo

Takeuchi, Hideyuki

Takeuchi, Yasu

Takeuchi, Yuko 
De la Monte, Suzanne

De la Serrana, Daniel G.

De Lange, Pieter

De Levie, Robert

De Lorenzo, Antonino

De los Santos Valladares, L.

De Lourdes Pereira, Maria

De Pedro, Nuria

De Peppo, Giuseppe M.

De Polo, Anna

De Steur, Hans

De Vendittis, E.

De Visser, S.P.

Dealy, Caroline N.

Dean, Zachary S.

Deans, Andrew J.

Dearden, John C.

DeCarlo, Arthur A.

Decker, Brian S.

Dedoussis, George V.

DeFilippis, Victor R.

Degregorio, Michael W

Deharo, Eric

Dehouck, Yves

Dekker, Marloes

Del Carpio-Perochena, A.

Delerue, Fabien

Deleuze, Michael

Delgado Olguín, Paul

Delivoria-Papadopoulou, M.

Demir, Ihsan Ekin

Demmerle, Justin

Demorrow, Sharon

Demyanets, Svitlana

Den Dunnen, Wilfred F.A.

Den Hertog, Jeroen

Denizot, B.

Denoyer, Delphine

Denti, Michela

Depalma, Ralph G.

Derks, Terry G.J.

Deshayes, Sébastien

Desideri, Alessandro

Desmarets, Christophe

Desmet, Tom

Dettori, Maria Luisa

Deurwaerdère, Philippe

Devergne, Odile

Devesa, J.

Dewi, Mewahyu

Dey, Moul

Dhandapani, Krishnan M.

Dhawan, Sangeeta
Lombardi, Pietro

Lonardo, Amedeo

London, Nir

Lonez, Caroline

Long, Audrey

Longley, D.B.

Loo, Ruey-Leng

Lood, Christian

López-Atalaya, José P.

Lopez-Hellin, Joan

Lopez-Llorca, Luis

Lopez-Vaamonde, Carlos

Lord, Megan S.

Lotrich, Francis

Lötsch, Jörn

Lou, Bih-Show

Lou, Jie-Chung

Lou, Xiang-Yang

Louime, Clifford

Loura, Luís M.S.

Løvdal, Trond

Lovell, Mark

Lu, Chi-Yu

Lu, Hong

Lu, Jian

Lu, Jinjian

$\mathrm{Lu}$, Jun

$\mathrm{Lu}$, Linchao

Lu, Ming-Wei

$\mathrm{Lu}$, Paul

Lu, Rong-Rong

Lu, Tse-Min

$\mathrm{Lu}, \mathrm{W}$

Luben, Tom

Lucassen, Paul J.

Lucchin, Margherita

Luco, Reini F

Ludwig, Roland

Ludwig-Müller, Jutta

Lui, Ed

Lui, Vincent Chi-Hang

Lum, Julian J.

Luna, Aurelio

Luna, Diego

Lunel-Fabiani, Françoise

Luo, Peigao

Luo, Zhong-Cheng

Lupiáñez, José A.

Luthey-Schulten, Zaida

Luzhkov, Victor B.

Lyng, Fiona M.

Lyng, Heidi

Lysaght, Joanne
Takumi, Shigeo

Takuro, Nakayama

Talbert, Joey N.

Tamura, Masaaki

Tan, Dun-Xian

Tanabe, M.

Tanabe, Yoo

Tanaka, Hiromi

Tanaka, Hiromitsu

Tanaka, Hiroyuki

Tanaka, Kohichi

Tanaka, Minoru

Tanaka, Naoki

Tanaka, Naonobu

Tanaka, Takuji

Tanaka, Tim Hideaki

Tanavde, Vivek

Tang, Wei

Tang, Wenlong

Tang, Xiaojia

Tani, Tokio

Tanigawa, Tetsuya

Tanimizu, Naoki

Tanner, N. Kyle

Tanoi, Keitaro

Tanonaka, Kouichi

Tanou, Georgia

Tao, Lin

Tao, Yaxiong

Tarantino, Giovanni

Tarozzi, Andrea

Tarro, Giulio

Tartaglia, Gian Gaetano

Tasciotti, Ennio

Taskinen, Seppo

Tate, Rothwelle J.

Tatsumi, Kazuya

Tatullo, Marco

Taupitz, Matthias

Tava, Aldo

Tavazzi, Barbara

Tawata, Shinkichi

Tayabali, Azam

Taylor, Larry $\mathrm{T}$.

Taylor, Maureen E.

Taylor, Molly A.

Taylor, Nicolas L.

Taylor, Peter W.

Taylor, Richard W.

Tazaki, Hiroyuki

Tchapla, Alain

Tcholakova, Slavka

Teeri, Teemu H. 
Dhondt-Cordelier, S.

Di Cesare Mannelli, L.

Di Giulio, Massimo

Di Luccia, Aldo

Di Paolo, Thérèse

Di Primio, R.

Di Renzo, L

Di San Lio, Gaetano M.

Di, Rong

Diamond, Betty

Dias, Carlos

Díaz, José

Diccianni, Mitchell B.

Díez, David

Dijkstra, Bauke W.

Dikalova, Anna

Ding, Yuchuan

Dinh, Dzung H.

Dini, Luciana

Dinis, Teresa

Diot, Christian

Dioum, Elhadji M.

Dittrich, Marcus

Dixon, Dan

Dixon, David A.

Diz, Debra I.

Dmitriev, Petr

Do, Yi-yin

Dobashi, Yoh

Dobens, Leonard L.

Doerr, Markus

Doerrler, William

Doi, Hideyuki

Dolinsky, Vernon W.

Domingos, Pedro M.

Dominguez, Gustavo A.

Donaghue, Jacqui

Dondi, Daniele

Doniach, Sebastian

Donnelly, Ryan F.

Donno, Dario

Donohoe, Bryon $S$.

D'orazio, John

Dorea, Jose

Dosio, Franco

Dosztányi, Zsuzsanna

Douglas, Jack F.

Douglas, Timothy E.L.

Doulias, P.-T.

Douni, Eleni

Dowd, Patrick F.

Doyle, Siamsa

Drakoulis, Nikolaos
Ma, Chaozhi

Ma, Daqing

Ma, Dik-Lung

Ma, Guojia

Ma, Jin Yeul

Ma, Liang

Ma, Wei

Ma, Yongjie

Ma, Yuelong

MacDiarmid, Colin W.

Mace, Thomas

Machiels, Kathleen

Macias Sanchez, Antonio J.

Macías, Pedro

MacIntosh, Gustavo C.

MacManes, Matthew

MacPhee, Iain A.M.

Macqueen, Daniel J.

Madej, Thomas

Maeda, Tatsuya

Maehr, René

Maestro, Miguel

Magata, Yasuhiro

Maggioni, Daniela

Magid, Abdulmagid A.

Magnaldo, Thierry

Magnus, Per

Magyari, Melinda

Mahasenan, Kiran

Maher, Christopher A.

Maher, Pamela

Mahimainathan, Lenin

Mahjoub, M.-S.

Mahmoud, Soheil S.

Maicas, Sergi

Maire, Pascal

Maisch, Tim

Maitre, Michel

Majumder, Mousumi

Mak, Anselm

Mak, Nai K.

Maki, Kosuke

Makinodan, Manabu

Malaguarnera, Michele

Malavasi, Fabio

Malchiodi-Albedi, F.

Malde, Alpesh

Malemud, Charles J.

Malemud, Charles J.

Malheiro, Ricardo

Mallipeddi, Prema Latha

Malmsten, Martin

Mancuso, Peter
Teets, Nicholas M.

Tegeder, Irmgard

Tegler, G.

Tehranchian, $\mathrm{P}$.

Tehrani, Kourosch A.

Teijido, Oscar

Teissie, Justin

Tejero, Jesús

Ten Cate, Hugo

Tenbrock, Klaus

Téoulé, Evelyne

Terachi, Toru

Teran, Francisco J.

Terao, Kimio

Teschke, Rolf

Tesli, Martin

TESSARO, Davide

Testai, Fernando D.

Tetard-Jones, Catherine

Tetsu, Osamu

Tettamanti, Gianluca

Tevosian, Sergei G.

Tewari-Singh, Neera

Tfayli, Ali

Thaler, Lea

Tharakaraman, Kannan

Theile, Dirk

Thein, Tun-Linn

Theodoly, Olivier

Theodoropoulos, John

Thevelein, Johan M.

Thèvenod, Frank

Thibaudeau, Sebastien

Thilmony, Roger

Thiriet, Marc

Thoma, Christian

Thomas, Helen E.

THOMAS, Olivier

Thomas, Olivier P.

Thompson, Deborah J.

Thompson, Jennifer

Thompson, Mary Kathryn

Thompson-Snipes, L.

Thomsen, Gerald

Thor, Ann D.

Thorin-Trescases, N.

Thornburg, Kent

Thrasyvoulou, Andreas

Threlkeld, Steven

Tian, Xiaoyu

Tian, Yingfang

Tichauer, Kenneth

Tielens, Loius 
Dressel, Alexander

Driessen, Christoph

Drobizhev, Mikhail

Drosten, M. Drosten

D'Souza, Manoranjan

Duan, Yuyou

Duarte, Filipe V.

Duarte, Margarida

Duarte, T.L.

Dube, Dipak K.

Dübel, Stefan

Dubey, Mukesh K.

Dubey, Raghvendra K.

Dubielecka-Szczerba, P.

Dubini, Alexandra

Dubrova, Yuri

Duda, Katarzyna

Dudás, József

Dufès, Christine

Duhamel, Jean

Dumas, Renaud

Dumez, Sylvain

Dumon, S.

Dumont, Marc

Dunbar, Gary L.

Duncker, David

Dundas, Ian

Dunham, Shari

Dunn, Louise

Durand, Jean-Oliver

Dürrwald, Ralf

Dusso, Adriana

Dustin, Lynn B.

Dvornyk, Volodymyr

Dwivedi, Chandradhar

Dytfeld, Joanna

Dzamko, Nicolas

Dziubla, Thomas

Ebner, Nicole

Ebrahimi, Kourosh H.

Eckardt, Kristin

Eckl, K.M.

Eder, Iris E.

Efird, Jimmy

Efrat, Himon

Eftekharpour, Eftekhar

Eggert, Jullie

Eggler, Aimee

Eglin, David

Egloff, Ann Marie

Ehrhardt, Anja

Ehrlich, Hermann

Eid, Nabil
Mandell, Samuel Pierce

Manenti, Raoul

Manfredi, Giovanni

Mang, Thomas S.

Manga, Prashiela

Mangialasche, Francesca

Mannell, Hanna

Manning, Thomas

Manolson, Morris F.

Manoranjan, Branavan

Mansoori, G. Ali

Månsson, Alf

Mantero, Sara

Manuella, Rigano

Manukjan, Georgi

Manz, Rudolf Armin

Marasco, Daniela

Marc, Isabelle

Marcacci, Maurilio

Marcelli, Augusto

Marchenko, Olga

Marchesan, Silvia

Marchetti, Philippe

Marco, Carmen

Marcos, Mercedes

Marcu, Kenneth B.

Marcucci, Fabrizio

Maréchal-Drouard, L.

Marinaki, A.M.

Marino, Angela

Mariottini, Gianluigi

Marison, Ian

Markou, Giorgos

Marmiroli, Nelson

Marondedze, Claudius

Marques, Fernanda

Marques, Isabel Paula

Marques, Paula A.S.S.

Marsault, Eric

Marshall, Gailen D.

Marsillach, Judit

Martel, An

Martel, Fátima

Martel, S.

Martens, Penny

Martí, Sérgio

Martín, A.

Martin, F.

Martin, Julia

Martin, Kathleen A.

Martin, Sara L.

Martin, Todd M.

Martín-Aragón, Sagrario
Tijssen, Peter

Tikoo, Suresh K.

Tilkorn, Daniel-johannes

Timko, Michael Paul

Timmerman, Peter

Timmons, Lisa

Timmusk, Salme

Tiozzo, Stefano

Tirone, Felice

Titica, Mariana

Titorenko, Vladimir I.

Tivendale, Nathan D.

Tiwari, Sanjay

Tobajas, Montserrat

Tobiume, Kei

Tobon, Yeny A.

Tognetti, Vincent

Tognini, Paola

Tokars, Valerie L.

Toledano, Manuel

Tolmachev, Vladimir

Tomasch, Jürgen

Tomasi, Nicola

Tombini, M.

Tomiyasu, Hirotaka

Tomlinson, Gail E.

Tommassino, Massimo

Tomofuji, Takaaki

Tomonaga, Keizo

Tompa, Peter

Tonda-Turo, Chiara

Tong, Lingying

Tong, Stephen

Toniolo, Antonio

Tonks, Nick K.

Tonnabel, Jeanne

Topisirovic, Ivan

Tordjman, Sylvie

Tori, Motoo

Toropov, Andrey A.

TORRENS, Francisco

Torres, J. Antonio

Torres, Josema

Tortora, P.

Tosti, S.

Touraud, Didier

Touyz, Louis Z.G.

Tovit, Rosenzweig

Toyoda, Atsushi

Trabace, Luigia

Trabocchi, Andrea

Trabolsi, Ali

Trachtman, Howard 
Eisel, Ulrich

Elati, M.

Eleouet, Jean-François

El-Gendy, Ahmed A.

Elhanafi, Ahmad Omar

Elhendy, Abdou

Elisabetta, Donzelli

Eljaafari, Assia

Elke Holinski-Feder, Elke

Elkhayat, Ehab S.

Ellervik, Christina

Ellis, Ronald E.

Elosua, Roberto

Elshimali, Yahya I.

Emerson, Joseph

Emond, M. Patrick

Enari, Masato

Endersby, N.M.

English, Niall

Eo, Seong Kug

Eppard, Elisabeth

Epperly, Michael

Ericson, Marica B.

Eriksen, Niels Thomas

Ertani, Andrea

Escames, Germaine

Escolà-Gil, Joan Carles

Esguerra, Jonathan Lou S.

Espen, Rimstad

Espinosa-Urgel, Manuel

Essani, Karim

Esteban, Maria Angeles

Estelrich, Joan

Esteve, Jordi

Esteve-Romero, Josep

Esteves, Ana Cristina

Ethirajan, Anitha

Evans, Bradley S.

Evans, David R.

Evans, Joseph L.

Eve, David

Evensen, Øystein

Ewart, Kathryn Vanya

Exner, Thomas

Expert, Dominique

Eykyn, Thomas R.

Eynde, Jean J. Vanden

Ezura, Yoichi

Fabiani, Roberto

Fabio, Vianello

Facelli, Julio C.

Fadl, Amin

Fagerstedt, Kurt V.
Martín-Cordero, Carmen

Martinelli, Adriano

Martinelli, fillopo

Martinez, Anne-sophie

Martínez, Constantino

Martinez, Manuel

Martínez, Miguel A.

Martinez, Vicente

Martínez-García, Pedro J.

Martínez-González, José

Martinez-Orgado, J.

Martínez-Sánchez, Noelia

Martino, Sabata

Martins, L. Miguel

Martinusen, Dan

Maruf, Abdullah Al

Marverti, Gaetano

Marzocco, Stefania

Masaaki, Konishi Masaaki

Masaki, Hitoshi

Masci, Stefania

Mashek, Douglas

Masi, Antonio

Masoero, Giorgio

Mason, D.L.

Masotti, Andrea

Masquida, B.

Masters, Colin L.

Mastrangelo, Anna M.

Masuda, Haruchika

Masuda, Kiyoshi

Masuda, Michiaki

Matar, Samir F.

Matés, José M.

Matesic, Diane F.

Mateus, Catarina

Mateus, Nuno

Matile, Stefan

Matoba, Nobuyuki

Matos, Ana Rita

Matros, Andrea

Matsubara, Joanne

Matsubara, Keiichi

Matsugo, Seiichi

Matsui, Toshiro

Matsunuma, S.

Matsuoka, Daisuke

Matsuoka, Masato

Matsuoka, Shin-ichi

Matsusaki, Michiya

Mattioli-Belmonte, M.

Mattoo, Autar

Mattsson, Jim
Traktuev, Dmitry O.

Trendowski, Matthew

Trenfield, Melanie A.

Tretyakova, Natalia Y.

Trevisan, Andrea

Triguero, Isaac

Trindade, Helena

Tripathi, Prateek

Tripodi, Marco

Trischitta, Francesca

Trollmann, Regina

Trollope, Alexandra

Tromp, Gerard

Trott, Josephine F.

Trotta, Christopher R.

Trouillas, Patrick

Trovato, Guglielmo

Trucco, Massimo

Trujillo, Glenda

Tryfonidou, Marianna A.

Tsaftaris, Athanasios

Tsai, C.Y.

Tsai, Cheng-Fang

Tsai, Eing-Mei

Tsai, Fuu-Jen

Tsai, Henry P.

Tsai, Hui-Hsu Gavin

Tsai, Keng-chang

Tsai, Shau-Wei

Tsai, Shih-Chang

Tsai, Sientang

Tsakalof, A.

Tschon, Matilde

Tsikas, Dimitros

Tsiplakou, Eleni

Tsitsilonis, Ourania E.

Tsonis, Panagiotis A.

Tsuji, Petra

Tsuji, Shoji

Tsukiyama, Takuji

Tsunoda, Ikuo

Tsutsui, Shigeyuki

Tu, Shi-Ming

Tubaro, Cristina

Tuck, Astrud R.

Tudisco, Raffaella

Tuggle, Christopher K.

Tulliani, Jean-Marc

Tuma, Pamela

Tung, Bui Thanh

Tung, Tao-Hsin

Turco, Gianluca

Turiel, Maurizio 
Fahlman, Richard P.

Faísca, Patrícia

Falahati-Anbaran, M.

Falasca, M.

Fan, Xiucheng

Fang, Wenwen

Fang, Xiangling

Fanizzi, Francesco

Faoro, Franco

Faraloni, Cecilia

Farinos, H. Merle

Fariselli, Piero

Farren, Matthew

Fata, Giorgio La

Faul, Christian

Fazio, Pietro Di

Fedele, Francesco

Fehling, Heather

Fei, Likun

Feichtinger, Georg A.

Feinendegen, Ludwig

Feizi, Soheil

Felby, Claus

Felder, Eduard

Feliers, Denis

Felix Saavedra, Maria José

Felli, Isabella C.

Felmlee, Daniel J.

Feng, Changjian

Feng, Dan

Feng, Xiqiao

Feng, Yangbo

Feng, Yibin

Feng, Z. Vivian

Fenoglio, Chiara

Fenoll, Carmen

Fenwick, Mark A.

Feo, Francesco

Feola, Mauro

Ferlizza, Enea

Fernandes, Emanuel M.

Fernandes, Susana C.M.

Fernandez, Jessie

Fernández, José J.

Fernández-Bolaños, Juan

Fernandez-Carvajal, Asia

Fernandez-Garcia, Marta

Fernandez-Lafuente, R.

Fernández-Ponce, M.T.

Fernández-Ruiz, Javier

Feron, Krishna

Ferraioli, Giovanna

Ferrand, Jonathan
Matyas, Jessica

Maunoury, V.

Mavragani, Clio P.

Mavrogonatou, Eleni

Mayer, Clemens

Mayhan, William G.

Maytin, Edward V.

Mazière, Cécile

Mazzucotelli, Elisabetta

McArdle, Harry

McArthur, J. Vaun

Mccarthy, John J.

McClung, C.A.

Mccullough, Michael

McCullumsmith, R.E.

McCully, James D.

Mccully, Kilmer S.

McDonagh, Brian

Mcdonald, Courtney

Mcdougall, Gordon J.

McFarland, Sherri

McGrath, Patrick T.

Mcgrew, Michael J.

McGuffin, Liam J.

McHenry, Peter

Mcintosh, E. David G.

Mcintosh, Robert

McKinney, Garrett J.

McLachlan, Gerry

McLaughlin-Drubin, M.

McMahon, Hilary E.M.

McMahon, Lawrence P.

McMahon, Peter

Mcmurray, David N.

McNamara, Coleen A.

Medeiros-Domingo, A.

Medina Piles, Vicente

Medina, Francisco Javier

Meeker, Rick B.

Meharg, Caroline

Mehboob, Shahila

Mehdizadeh, Mehdi

Mehr, Shaadi

Mehta, Akul Y.

Meijles, Daniel

Meiser, Peter

Melendy, Tom

Mélinon, Patrice

Melkani, G.C.

Mells, Jamie E.

Melo, Eduardo P.

Meloni, Rolando

Melzig, Matthias
Turkbey, B.

Turng, Lih-Sheng

Turunen, Mikko P.

Tuttolomondo, Antonino

Tyagi, Neetu

Tyner, Jeffrey W.

Typas, Milton A.

Tyurina, Yulia Y.

Tyynismaa, Henna

Tyystjärvi, Taina

Tzang, Bor-Show

Tzetis, Maria

Ubertini, Filippo

Uchida, Shizuka

Uchida, Yoshikazu

Ueda, Natsuo

Uegaki, Koichi

Uehara, Tomoya

Ueno, Shu-ichi

Ufer, Christoph

Uhal, Bruce D.

Uhde-Stone, C.

Ulisse, Salvatore

Ullah, Ashik

Ullah, Hemayet

Umemura, Myco

Umemura, Shigeki

Uppugunduri, C.R.

Urabe, Daisuke

Urbanc, Brigita

Ureña, Enric

Ushio-Fukai, Masuko

Utashima, Yuu

Uus, Kai

Uversky, Vladimir

Uversky, Vladimir N.

Vacca, Angelo

Vago, Riccardo

Vaira, Valentina

Vaisar, Tomáš

Vaisburg, Arkadii

Vajro, Pietro

Vale, Paulo

Valencia, C. Alexander

Valencia, Gregorio

Valenciano, Ana Isabel

Valenzuela, Manuel

Valero, Rosendo

Valli, Veronica

Valone, Steven M.

Valtueña, Francisco J.

Valverde, Ángela M.

Van Coppenolle, Fabien 
Ferrante, Antonio

Ferrarese, Carlo

Ferrarini, Alberto

Ferrazzi, Enrico

Ferreira, A.F.

Ferreira, Daniel

Ferreira, João R.

Ferreira, Thierry

Ferrini, Silvano

Fetzner, Susanne

Fielding, Joanne

Fierabracci, Alessandra

Findlay, Victoria J.

Finelli, Carmine

Fiocco, Daniela

Fiona, Lynch

Fiorina, Paolo

Firer, Michael

Firestone, Gary

Fischer, Hans-Martin

Fitton, Helen

Fitzgerald, Rebecca C.

Flisikowska, Tatiana

Florea, Ana-Maria

Florio, Tullio

Flower, Andrew

Fluhr, Robert

Flynn, Aidan

Foderà, Vito

Fomenko, Dmitri E.

Fondevilla, Sara

Forbes-Hernandez, T.Y.

Ford, Lance P.

Forest, Jean-Claude

Forhead, Alison J.

Formisano, Pietro

Forster, Britta

Forthun, Rakel Brendsdal

Foster, Leonard J.

Fosu-Nyarko, John

Foti, Mario C.

Fotopoulos, Vasileios

Fouilland, Eric

Foulds, Charles

Fournier, Claudia

Fox, Keith R.

Francino, Pilar

Francis, Gordon

Franco, Diego

Frandsen, Henrik Lauritz

Frank, Saša

Franklin, Gregory

Franklin, Michael J.
Memelink, Johan

Mencherini, Teresa

Mendes, Eduarda

Mendoza-Cózatl, D.G.

Menendez, Rosa

Meng, Xiangbing

Meng, Yizhi

Ménoret, Séverine

Mentzer, Steven J.

Mercer, Carol

Mercer, Julian F.B.

Merkel, Olaf

Merlino, Antonello

Merlot, Sylvain

Metheny-Barlow, Linda J.

Metherell, Louise

Metzinger, Laurent

Meyer, Barbara J.

Meyer, Denise

Meyer, Etienne

Meyer, Florencia

Meyer, Hans-Peter

Meyerholz, David

Mezzenga, Raffaele

Miao, Jun

Micali, Norberto

Miccadei, Stefania

Micheau, Olivier

Michelhaugh, Sharon K.

Michels, Alexander J.

Michetti, Fabrizio

Michiels, Carine

Mico, Juan A.

Midde, Krishna

Midgley, Adam

Mielenz, Manfred

Migita, Satoshi

Migliaccio, Anna Rita

Migliore, Lucia

Miguel, Graça

Mikami, Toshinari

Miki, Yasuhiro

Milà, Montserrat

Milde-Langosch, Karin

Mildner, Michael

Milella, Luigi

Milhinhos, Ana

Millay, Douglas P.

Millecamps, Magali

Milledge, John J.

Miller, Aaron W.

Miller, Donald W.

Miller, Ingrid
Van Dam, Mike

Van Damme, Jo

Van de Donk, Niels W.C.J.

Van De Sluis, Bart

Van Den Bergh, Hubert

Van den Meiracker, A.H.

Van den Oord, Stijn C.H.

Van der Kuyl, Antoinette

Van der Lee, Robin

Van Der Meer, Peter

Van Der Straaten, Tahar

Van Der Voet, Gijsbert

Van Duijn, Bert

Van Dyke, Knox

Van Dyke, Mark E.

Van Kessel, Ad Geurts

Van Lieshout, Lisette

Van Oers, Nicolai

Van Veldhuizen, Peter

Van Vliet, Sandra J.

Van Wolfswinkel, J.C.

Vanakker, Olivier

Vanamala, Jairam

Vanarotti, Murugendra

Vandamme, Dries

Vandenbussche, Filip

Vanella, Luca

VanHorssen, Jack

Van't Hof, Arnoud W.J.

Vantarakis, Apostolos

Vaporidi, Katerina

Varela-Ramirez, A.

Varghai, Davood

Varra, Michela

Varrot, Annabelle

Vashee, Sanjay

Vasiljevic, Todor

Vassalle, Cristina

Vassilopoulos, George

Vaudry, David

Vaughan, Gilberto

Vaughan, Roger A.

Vavvas, Demetrios G.

Vaz, Deisi Altmajer

Vecchione, Carmine

Vecoli, Cecilia

Vedani, Angelo

Veenstra, Jan A.

Veeranki, Sudhakar

Velasco Ortega, Eugenio

Velez, Zélia

Velluto, Lucia

Veloso, Fernando T. 
Franklin, Rima B.

Frankowski, Marcin

Franssen, Frits

Franssen, Maurice C.R.

Franzke, Claus-Werner

Frauman, Albert G.

Freedman, Mark Steven

Freije, José M.P.

Freitas, Ana C.

Frenking, Gernot

Fresta, Massimo

Freund, Dana

Freund-Levi, Yvonne

Friák, Martin

Fribley, Andrew M.

Fridman, Gregory

Friedmann, Anton

Froeyen, Matheus

Fröhlich, Eleonore

Frolov, Andrej

Frost, Sofia

$\mathrm{Fu}$, Shu Man

Fujii, Takao

Fujimori, Ko

Fujita, Masayuki

Fujita, Naoko

Fujita, Tetsuji

Fujita, Yuichi

Fujiwara, Hironori

Fujiwara, Ryoichi

Fujiyama, Kazuhito

Fukagawa, Tatsuo

Fukoura, Hirotaro

Fukui, Hiroko

Fukunishi, Yoshifumi

Fuller-Espie, Sheryl L.

Fulzele, Sadanand T.

Furneri, Pio Maria

Fuso, Andrea

Gaerstel, Matthias

Gaiddon, C.

Gailer, Jürgen

Gaitzsch, Jens

Gaj, Thomas

Gal, A.

Galanakis, Charis

Galeone, Antonio

Galibert, Francis

Galli, Andrea

Gallo, Antonio

Galvão, Adelino M.

Gálvez, Julio

Galvez-Valdivieso, G.
Miller, Jessica

Miller, Vandana

Mills, David

Mills, Kenneth V.

Milosevic, Ana

Min, Jun-Ki

Minagar, Alireza

Minami, Takashi

Minamino, Tetsuo

Minardi, Daniele

Minehan, Thomas G.

Minella, Alex

Minocha, Subhash C.

Minuk, Gerald

Miranda, Carlos

Mirande, Marc

Mirzayans, Razmik

Mitani, Akio

Mitra, Abhisek

Mittal, Sandeep

Miura, Yoshiko

Miyamoto, Hiroshi

Miyamoto, Kei

Miyamoto, Koji

Miyamoto, Takeshi

Miyaoka, Hiroaki

Miyata, Jun

Miyazaki, Teruo

Miyoshi, Daisuke

Mizuguchi, Mineyuki

Mizuno, Tetsuya

Mizuta, S.

Mo, Yin Yuan

Moats, Rex A.

Moazedi-Fuerst, F.

Mocanu, G.

Mocchegiani, Eugenio

Mocellin, Simone

Mockaitis, Keithanne

Modirrousta, Mandana

Modugno, Francesca

Mognato, Maddalena

Mohammad, Ramzi M.

Mohammadi, Alireza M.

Mohanam, Sanjeeva

Mohanta, Tapan

Mohr, Fabian

Moioli, Bianca

Molasiotis, Athanasios

Moldzio, Rudolf

Molina, Juan Jesus

Molina-Bolívar, J.A.

Møller, Ian M.
Veloso, Javier

Venier, Paola

Venturini, Danielle

Venukadasula, Phanindra

Verardo, Vito

Verburg, Frederic

Verderio, Claudia

Verderio, Paolo

Verdolotti, Letizia

Vergara, Alessandro

Vermeulen, Louis

Vernhettes, Samantha

Verron, Elise

Verstraelen, Toon

Vetvicka, Vaclav

Viana Baptista, Pedro

Viappiani, Cristiano

Vicente, Cláudia S.L.

Vickaryous, Matthew K.

Vickers, Mark

Vidic, Jasmina

Viehweger, Katrin

Vieira, Cristina P.

Vieira, Helena L.A.

Viggiano, Davide

Vilagran, I.

Vilke, Gary M.

Villacorta, Luis

Villanneau, Richard

Villari, Valentina

Villena, Josep A.

Viluksela, Matti

Vincent, Stéphane D.

Viola, Giampietro

Viscomi, Maria Teresa

Visser, Lydia

Vistoli, Giulio

Vitali, Jacqueline

Vitali, Matteo

Vitetta, Luis

Vizan, Pedro

Vladimirov, Vladimir I.

Vogel, Ulla

Vogl, Thomas

Volbracht, Christiane

Von Delius, Max

Von Kalm, Laurence

Von Knethen, Andreas

Von Szentpály, László

Vondrasek, Jiri

Vonk, L.A.

Voronov, Irina

Voss, Randal 
Galzitskaya, Oxana V.

Gamal, Nesrine

Gambardella, Antonio

Gambino, G.

Gammone, M.A.

Gams, Walter

Gan, Wenjian

Ganguli-Indra, Gitali

Ganini, Douglas

Ganta, Chanran K.

Gao, Liangliang

Gao, Xiaohua

Gao, Xue

García Morote, F.A.

García, Tania

García-Caballero, Melissa

Garcia-Moreno, Diana

Garcia-Orad, Africa

García-Osorio, Cesar

Garcia-Salcedo, Jose A.

Gard, Paul

Gardner, Andrew W.

Gardner, Peter J.

Garg, Parveen K.

Gargiulo, Gaetano

Garrabou, Glòria

Garriga, Pere

Gary-Bobo, Magali

Gasque, Philippe

Gatti, Laura

Gayral, Philippe

Gazdhar, Amiq

Gazouli, Maria

Ge, Yubin

Geerts, Hugo

Geiser, Dawn

Geisler-Lee, Jane

Geissler, Nicole

Gelmann, Edward

Genovese, Giuseppa

Gentile, Piergiorgio

George, Kimberly S.

Georgiou, Ioannis

Gepts, Paul

Ger, T.R.

Geraci, Fabiana

Geraldes, Pedro

Gerhard, Markus

Gerke, Jörg

Germain, Doris

Germershaus, Oliver

Gershburg, E.

Gershon, Timothy
Molvarec, Attila

Molyneux, Karen

Monaco, Edward A., III

Monji, Akira

Monk, Jennifer

Monsees, Thomas

Montagut, Clara

Monteil-Rivera, Fanny

Monteiro Ramalhinho, A.C.

Montesinos-Pereira, D.

Monti, Daniela

Monti, Maria Chiara

Montoliu, Lluis

Moon, Hyung-In

Moon, Il Soo

Moon, Jisook

Moon, Young-Ah

Morales, María Belén L.

Morales, Patricia

Moran, Corey S.

Morandi, Luca

Morbidelli, Massimo

Moreau, Regis

Moreira, J.B.N.

Morell, Christophe

Morello, Roy

Moretti, Marcelo L.

Morgan, Claire

Morgan, William

Morgante, Francesca

Mori, Hitoshi

Moriguchi, Takaya

Morikawa, Hisao

Morikis, Dimitrios

Morillon, R.

Morishita, Ryuichi

Morita, Kyoji

Mörl, Mario

Mormile, Raffaella

Moro, Loredana

Morone, Piergiuseppe

Morotti, Alessandro

Möröy, Tarik

Morris, Brian J.

Morris, Dylan

Morris, John

Morris, Matthew

Morris, May C.

Morrison, Douglas

Morsczeck, Christian

Mort, John S.

Mortier, Jérémie

Moseke, Claus
Voz, Marianne L.

Wachten, Dagmar

Wada, Jun

Wagner, Amy K.

Wagner, Anika

Wagner, Brent

Wagner, Carol

Wagner, David

Waheed, Abdul

Waite, Matthew M.

Wakabayashi, Ken-ichi

Wakino, Shu

Walker, Celia

Walker, David

Walker, Lary C.

Wallace, G.

Wallace, Maeve E.

Wallentin, Lars

Wallhäusser-Franke, E.

Walsh, David

Walther, Dirk

Wambach, Jennifer

Wan, Jian-bo

Wan, xiaochun

Wanasundara, Janitha

Wandosell, F.

Wang, Bo-Cheng

Wang, Chin-Kun

Wang, Chunling

Wang, Dong

Wang, Fengshan

Wang, Haibo

Wang, Han-Ching

Wang, Huizhi

Wang, Jian

Wang, Jian-Wen

Wang, Jianying

Wang, Jinhu

Wang, Junjian

Wang, Junjie

Wang, Kai

Wang, P.

Wang, Peng

Wang, Qun

Wang, Rui

Wang, Ruoxiang

Wang, Timothy C.

Wang, Tsung-Shing

Wang, Tzu-hao

Wang, Wenxin

Wang, Xiang Simon

Wang, Xiaohong

Wang, Xinkun 
Geuna, Stefano

Gewirtz, David A.

Ghanotakis, Demetrios F

Gharbi, Y.

Ghelardi, Emilia

Ghersi, Dario

Ghiladi, Reza A.

Ghoraani, Behnaz

Ghose, Ranajeet

Giaginis, Constantinos

Giampiei, Francesca

Giannaccini, Martina

Giannattasio, Sergio

Giaouris, Efstathios

Gibot, Laure

Giera, Martin

Giesbrecht, Gerald F.

Gilbert, Nick

Gilch, Sabine

Giles, Wayne R.

Gill, Chris Ir

Gillings, Michael

Gilson, Michael

Giol, Elena Diana

Giordano, Antonio

Giovarelli, Mirella

Girao, Henrique

Giridhar Babu, A.

Girón-González, J.-A.

Gisselmann, Günther

Giuliani, Daniela

Gjini, Evisa

Glauser, Gaetan

Gleich, Gerald J.

Glembotski, Christopher C.

Glocker, Erik-Oliver

Gloria, Antonio

Gnana-Prakasam, Jaya

Goberdhan, Deborah CI

Gobinet, Cyril

Godin, Bruno Godin

Goergen, Craig J.

Goglia, Fernando

Gojo, S.

Goldberg, Inna Khozin

Golding, Jon P.

Goldman, Myla D.

Goldoni, Matteo

Goldring, Mary

Goldstein, Aaron S.

Goldstein, Benjamin I.

Goldup, Stephen

Golestaneh, Nady
Moshaverinia, Alireza

Moss, Alan C.

Mota, Manuel

Mott, Justin L.

Motterlini, Roberto

Mou, Chung-Yuan

Moulis, Jean-Marc

Mouradov, Aidyn

Moxon, Joseph V.

Mudaliar, Sunder

Mudalige, Thilak K.

Mueller-Roeber, Bernd

Muir, Kenneth

Muise-Helmericks, R.C.

Mukaida, Naofumi

Mukherjee, Tusharmouli

Mukhopadhyay, Partha

Mukudai, Yoshiki

Müller, Dafne

Muller, Sylviane

Müller-Buschbaum, Peter

Mulner-Lorillon, Odile

Munang'andu, Hetron M

Munoz, Macarena

Muñoz-Torres, Manuel

Munshi, Nikhil V.

Muraguchi, Hajime

Murai, Koji

Murakami, Kazuma

Murakami, Yasufumi

Murashita, Koji

Murata, Yoshiyuki

Muroi, Carl

Murono, Shigeyuki

Murray, Aja

Murray, Brent W.

Murray, Christopher J

Murray, James D.

Murray, Megan

Murray, Patricia

Murrell, Dédée F.

Murtas, Daniela

Murugaiyan, Jayaseelan

Muschel, Ruth J.

Musumeci, Giuseppe

Musumeci, Teresa

Muthusamy, Visalini

Muto, Tomoyuki

Mutoh, Tatsuro

Muylaert, Koenraad

Muyldermans, Serge

Muzzarelli, Riccardo A.A.

Myklebost, Ola
Wang, Xungai

Wang, Ya

Wang, Yadong

Wang, Yan-Hsiung

Wang, Ying

Wang, Ying-Jan

Wang, Yonghua

Wang, Yuechun

Wang, Yun-Ming

Wang, Zhang

Wang, Zhiguang

Wang, Zhong Q.

Wanke, Dierk

Warby, Simon C.

Ward, Peter A.

Warren, Gregory L.

Warzecha, Tomasz

Wasmuth, Susanne

Watanabe, Masakatsu

Watanabe, Masatoshi

Watanabe, Toru

Watanabe, Toshio

Watanabe, Yuichiro

Watashi, Koichi

Wätjen, Wim

Watson, Christa

Weathers, Palmela J.

Webb, I.C.

Weber, Franz E.

Weber, J. Mark

Weber, Jost

Webster, Marie R.

Weese, Scott

Wegiel, Barbara

Wei, Guor-Jien

Wei, Jing

Weigand, Annika

Weigand, Julia E.

Weiler, Hartmu

Weiss, Julia

Weiss, Norbert

Weitzel, Joachim M.

Wen, C.M.

Wen, Zhiyou

Weng, Ching-Feng

Wenger, Nanette K.

wenlong, Chang

Werling, Dirk

Werner, Rudolf A.

Wertz, Philip

Westendorf, Johannes

Wetterberg, Lennart

Wheeler, Thomas 
Gollasch, Stephan

Golledge, Jonathan

Goltsov, Alexey

Goltz, Diane

Gomer, Richard H.

Gomes, Helder T.

Gómez, Gustavo

Gómez-Jeria, J.-S.

Gómez-Laguna, J.

Gonçalves, Ana Luísa

Gonçalves, António Pedro

Gonçalves, Olivier

Gondhalekar, Ameya D.

Gondi, Christopher S.

Góngora-Castillo, Elsa

Gonzales, Eric

Gonzalez Muniesa, Pedro

González, María Pilar

Gonzalez, Ramon

González, Víctor M.

González-Bello, C.

González-Díaz, H.

González-Maeso, Javier

Goossens, N.

Gordon, Catherine A.

Gorgoulis, Vassilis G.

Gori, Tommaso

Gorinstein, Shela

Gorman, Shelley

Goswami, A.

Gothilf, Yoav

Goto, Kazuo

Goto-Inoue, Naoko

Gotor-Fernández, Vicente

Gott, Ryan C.

Gottesman, Michael M.

Goulão, Luís Filipe S.

Gould, Gwyn

Gourrierec, José Le

Govindarajulu, R.

Gowans, Gordon

Grabrucker, Andreas

Graca, Luis

Gracias, David

Grady, Bart P.X.

Graether, Steffen P.

Graham, Ann

Graham, Neil

Grammatikakis, Ioannis

Gramzow, Lydia

Granata, Simona

Graner, Michael

Granitzer, Petra
Myung, Pyung-Keun

Nadal, Pol

Naegele, R.P.

Nag, Dilip K.

Nagai, Kouhei

Nagano-Takebe, Futami

Nagasawa, Hiromichi

Nagata, Takeshi

Nagatomo, Shigenori

Nagy, Istvan

Nahashon, Samuel

Nair, Ashwin

Nair, Lakshmi S.

Naito, Ken

Naito, Yuji

Najimi, Mustapha

Naka, Hiroaki

Naka, Tetsuji

Nakagawa, Yusuke

Nakai, Masaaki

Nakaki, Toshio

Nakamoto, Shingo

NAKAMURA, Kazuhiko

Nakamura, Kazuki

Nakamura, Kozo

Nakamura, Noriko

Nakao, Minoru

Nakashima, Kazuo

Nakatani, Yoshihiko

Nakayama, Koh

Nakayama, Yasumune

Nakayama, Yuji

Namihira, Masakazu

Nanashima, Atsushi

Nånberg, Eewa

Nandrot, Emeline F.

Narayana, Ashwatha

Narod, Steven

Nasrallah, June B.

Nasri, Rim

Natarajan, Sathish Kumar

Natesan, Senthil

Naughton, Patrick

Naumann, Todd A.

Naumova, Anna V.

Navarra, Michele

Navarro, Miguel

Naviglio, Silvio

Naya, Francisco J.

Nazar, Ross N.

Neacsu, Madalina

Neerinckx, Barbara

Negi, Surendra S.
Whisner, Corrie

White, Jason

Whiteside, Theresa L.

Whitford, Paul C.

Wiborg, Ove

Widhalm, Georg

Widhalm, Kurt

Wiechec, Emilia

Wiegertjes, Geert

Wielstra, Ben

Wildemann, Britt

Wilharm, Gottfried

Wilkinson, J.M.

Williams, David C., Jr.

Williams, Thomas L.

Wilson, Adam C.

Wilson, D.B.

Wilson, Karl A.

Wilson, Michael

Wilson, Robert

Wiltshire, Esko J.

Wimmers, K.

Wingert, Rebecca A.

Winkler, G. Sebastiaan

Winkler, Thomas

Winship, Ian R.

Winssinger, Nicolas

Winterbone, Mark

Wirth, D.

Wischhusen, Joerg

Witt-Enderby, Paula

Witzel, Katja

Woeltje, Michael

Woisel, Patrice

Wold, William S.M.

Wolf, Jennifer Moriatis

Wolfl, C.

Wolfson, Adi

Woloszyk, Anna

Won, Chong Hyun

Wong, Albert H.C.

Wong, Anderson On Lam

Wong, Boon-Seng

Wong, Chi-Ming

Wong, K.-K.

Wong, Kenneth K.Y.

Wong, Melanie

Wong, Vincent Kam Wai

Woo, Hee Chul

Woo, So-Youn

Wood, W. Gibson

Woodfield, Tim

Woodfield, Tim B.F. 
Grant, Wiliam

Grassi, Gabriele

Grattagliano, Ignazio

Gray, Neil D.

Graziano, Giuseppe

Graziano, Guella

Grebien, Florian

Green, Charlotte

Greenberger, Joel S.

Greene, Lesley H.

Greenidge, P.A.

Greening, David W.

Greenwood, David

Greenwood, Michael T.

Greim, Helmut

Gresshoff, Peter

Grey, Carl

Grieco, Carmine R.

Grieco, Francesco

Griese, Matthias

Grignani, G.

Grimholt, Unni

Grimley, Philip M.

Grimpe, Barbara

Grinberg, Daniel

Grinfeld, Jacob

Gris, Jean Christophe

Grisoni, Francesca

Groenendyk, Jody

Gromak, Natalia

Gros, Frédéric

Grosso, Clara

Grosso, Giuseppe

Gruh, Ina

Grusch, M.

Gsponer, Jörg

$\mathrm{Gu}$, Hong-chen

$\mathrm{Gu}$, Jian

$\mathrm{Gu}$, Yan

Guaricci, Andrea Igoren

Gudiña, Eduardo

Gueimonde, Miguel

Guénal, I.

Guentsch, Arndt

Guerra, Davide

Guerre-Millo, Michèle

Guerzoni, Maria E.

Guglielmi, P.O.

Guillaume, Sophie

Guilliams, M.

Guillot, Bertrand

Guin, Sunny

Gulbinsh, Erich
Negro, Enrico

Neier, Reinhard

Neirinckx, Virginie

Nellaiappan, K.

Nemunaitis, John

Nesmelov, Yuri E.

Neugart, Susanne

Neuhauss, Stephan

Neutzner, Albert

Neuzil, Jiri

Nguyen, Phuong

$\mathrm{Ni}$, Weiming

Nicassio, Francesco

Nichols, A.R.L.

Nicholson, Allen W.

Nicolás, Francisco E.

Niculescu, Mihai D.

Niel, Ed van

Niemann, Bernd

Nieminen, Anna-Liisa

Nigatu, Yeshambel T.

Niidome, Takuro

Niimi, Tomoaki

Niino, Masaaki

Nijnik, Anastasia

Nikamo, Pernilla

Nikolakakis, Ioannis

Nikolic, Dejan

Nilsson, Per

Ninfali, Paolino

Ning, Baitang

Niranjan, Rituraj

Nishida, Yoshihiro

Nishihara, Tatsuji

Nishina, Hiroshi

Nishio, Zenta

Nishiwaki, Hisashi

Nishiyama, Toshio

Nisnevitch, Marina

Nissilä, Marika E.

Nistala, Ravi

Nkeng-Efouet, Pepin A.

Nobili, Stefania

Noguera, Daniel R.

Noh, Hyunjin

Nojiri, Takashi

Nomoto, Shuji

Nomura, $\mathrm{M}$.

Nonami, Atsushi

Noordzij, Walter

Norman, Peter

Norman, Robert

Normand, Philippe
Woods, Matthew

Woody, Robert W.

Worth, Andrew

Wright, Louwrance

$\mathrm{Wu}$, Cheng-Hsun

$\mathrm{Wu}$, Chia-Wen

$\mathrm{Wu}$, Huey-Nan

$\mathrm{Wu}$, Jian-Ping

$\mathrm{Wu}$, Kenneth K.

$\mathrm{Wu}$, Keqiang

$\mathrm{Wu}$, Lianfeng

Wu, Ming-jiuan

$\mathrm{Wu}$, Mousheng

$\mathrm{Wu}$, Nicholas

$\mathrm{Wu}$, Pensee

$\mathrm{Wu}$, Ren-Jang

$\mathrm{Wu}$, Sheng-Chi

Wu, Shih-Hsiung

$\mathrm{Wu}$, Shu-Yii

$\mathrm{Wu}$, Tzong-Yuan

$\mathrm{Wu}$, Xiaoqing

$\mathrm{Wu}, \mathrm{Y}$.

$\mathrm{Wu}$, Yuliang

Wung, Being-Sun

Wursthorn, Karsten

Würtz, Morten

Wysocki, Lawrence J.

Wyss, Christine

Xiao, J.

Xiao, Li

Xiao, Zhousheng

Xie, Guofeng

Xodo, Luigi E.

$\mathrm{Xu}$, Baoshan

$\mathrm{Xu}$, Leyuan

Xuan, Xiangchun

Xuan, Zhenyu

Xue, Bin

Xue, Meilang

Xue, Xiang

Yada, Takashi

Yagi, Hiroshi

Yahubyan, Galina

Yamabe, Shinichi

Yamada, Sohsuke

Yamagishi, Akihiko

Yamaguchi, Hiroyuki

Yamaki, Koji

Yamamoto, Akihiro

Yamamoto, Masayuki

Yamamoto, Norio

Yamamura, Soichiro

Yamano, Koji 
Gulino, Rosario

Gunaje, Jayarama

Gunkel, Günter

Guo, Lin

Guo, Li-Tao

Guo, Min

Guo, Peixuan

Guo, Xing

Guo, Z. Sheng

Gupta, Ankit

Gupta, Sanjay

Gupta, Sudhiranjan

Gupta, Vivek K.

Gurevich, Eugenia V.

Gurevich, Vsevolod V.

Gursky, Olga

Gurzov, Esteban N.

Gutekunst, Kirstin

Guthridge, Mark A.

Gutiérrez Martín, S.

Gutschner, Tony

Guttery, David

Gyselaers, W.

Haapalainen, Minna

Haapasalo, Annakaisa

Haas, Hubertus

Haase, Hajo

Haass, Nikolas K.

Hackenberg, Michael

Hadjiargyrou, Michael

Hadjipavlou-Litina, D.

Hadwiger, Lee

Hadziyannis, Emilia

Haenen, Guido R.M.M.

Hagedoorn, Peter-Leon

Hagenacker, Tim

Hahn, Matthias

Hahn, Sei Kwang

Hai, Bo

Haider, Norbert

Hajnsdorf, Eliane

Haldrup, K.

Halford, Nigel G.

Halina, Machelska-Stein

Hall, Joseph C.

Hallegraeff, Gustaaf M.

Hallouard, François

Halonen, Sandra K.

Hamamura, K.

Hamann, Martine

Hamdy Roby, Mohamed H.

Hamerlynck, Erik P.

Hammond, John
Norrild, Bodil

Norval, Mary

Nothnick, Warren B.

Nouri, Keyvan

Nouri, M.-Z.

Novak, Johannes

Nowikovsky, Karin

Nuevo, Michel

Numakawa, Tadahiro

Nunney, Leonard N.

Nunzio, Mattia Di

Nussler, Andreas K.

Nylander, Karin

Nyström, Andreas M.

Nyström, Kristina

O'Carroll, Simon J.

O'Donnell, Kieran J.

Oakeson, Kelly F.

Oakley, Brian B.

Oancea, Elena

O'banion, Michael

Obare, Sherine

Obdeijn, M.C.

Oberholster, Anita

Ochiya, Takahiro

Ocio, Enrique

Oetting, William S.

Ogata, Tadanori

Ogata, Yorimasa

Ogawa, Noriko

Ogawa, Ryuichi

Ogawa, Tomohisa

Ogawa, Y.

Ogawa, Yuko

Ogi, Kazuhiro

Ogilvie, Caroline

Ogino, Shuji

O'Gorman, David B.

Ogra, Yasumitsu

Ogunjirin, Adebowale E.

Oh, Jae-Min

Oh, Sae-ock

O'Hara, Laura

Ohi, Kazutaka

Ohkohchi, Nobuhiro

Ohlendieck, Kay

Ohnishi, Masatoshi

Ohnuma, Tohru

Ohta, Takeshi

Ohtaki, Hirokazu

Okabayashi, Jun

Okawa, Masakazu

Okazaki, Toshiro
Yamauchi, Satoshi

Yamauchi, Takahiro

Yamaza, Takayoshi

Yan, Bowen

Yan, Jun

Yan, Liang Jun

Yan, Shan

Yang, Da-Qing

Yang, Eddy S.

Yang, Fan

Yang, Guang-dong

Yang, Hongshun

Yang, Jen-Chang

Yang, MinoYang

Yang, Pingfang

Yang, Seong Wook

Yang, Wei-hsiung

Yang, Weng-Lang

Yang, Xiaoyan

Yang, Yong

Yang, Yongguang

Yang, Yuedong

Yang, Yuzhe

Yano, Shozo

Yao, Izumi

Yao, Wei

Yao, Zhong-Ping

Yasuda, Kazuki

Yasuhara, Takao

Yasuko, Manabe

Yazlovitskaya, E.M.

Yeddula, Narayana

Yeh, Chau-Ting

Yeh, Jui-Ming

Yeh, Jwu-Lai

Yeh, Kuo-Chen

Yeh, Min-Long

Yellon, Derek M.

Yen, Feng-Lin

Yennu-Nanda, V.G.

Yeoman, Caarl

Yeung, Kam C.

Yeung, Sai-Ching Jim

Yezierski, Robert $\mathrm{P}$.

Yilmazel, Yasemin D.

Yin, John

Ying, Shao-Yao

Yokobori, Takehiko

Yokoi, Tsuyoshi

Yokota, Shinso

Yokoyama, Tomoyuki

Yokoyama, Yoshihito

Yonekura, Shinichi 
Hamrah, P.

Hamzeh, Mahsa

Han, Dong Wook

Han, Inn-oc

Han, Jin

Han, Quanbin

Han, Sang-bae

Han, Seung Hyun

Han, Yeon Soo

Hanagasi, Hasmet A.

Hanaoka, Mitsumasa

Hanawa, Haruo

Hanazono, Yutaka

Hanhineva, Kati

Hann, Hie-Won

Hannafon, Bethany

Hannan, Anthony J.

Hannestad, Jonas

Hanschen, Franziska S.

Hansen, T.F.

Harada, H.

Harada, Mamoru

Harada, Naoki

Harada, Yoshie

Haraguchi, Yuji

Haraldsen, Guttorm

Harari, Daniel

Harauz, George

Harb, Jamil

Hardingham, Jennifer E.

Hardtke-Wolenski, M.

Hardy, John George

Hardy, Margaret

Hare, Dominic J.

Hare, Joshua M.

Harn, Chee Hark

Harris, Jennifer W.

Harris, Linda

Harrison, Marcia A.

Harrison, Paul H.M.

Harrison, Roger

Hart, David A.

Hart, Ronald

Hartnett, David C.

Hartz, Kara E. Huff

Haruki, Mitsuru

Harvey, Richard

Hasanuzzaman, Mirza

Hasegawa, Tomonobu

Hasegawa, Yasushi

Haspel, Nurit

Hassan, Mohamed

Hassel, Bret
OKUMURA, Nobuaki

Okuyama, Hidetoshi

Oliveira, Andreia

Oliveira, P.A.

Oliviero, F.

Ollas, Carlos de

Ollikainen, Miina

Olson, Michael F.

Olson, Peter D.

Olsson, Christian

Oltra, Elisa

Ometto, F.

Ondruschka, Benjamin

Onozaki, Kikuo

Oono, Youko

Oostenbrink, Chris

Oosterkamp, Margreet

Oosterwijk, Egbert

Ordi-Ros, Josep

O'Reilly, Michael A.

Oresnik, Ivan J.

Orlova, Elena V.

Orner, Brendan P.

Ortiz, Gabriela Toledo

Ortiz, Inmaculada

Ortiz-Soto, Maria

Ortlund, Eric A.

Osada, Jesús

Osanai, T.

Oshitari, Toshiyuki

Oster, Henrik

O'Sullivan, Cathryn

Osumi, Kenji

Ota, Motonori

Otsuka, Fumio

Ottinger, Elizabeth A.

Oudit, Gavin Y.

Ouzounidou, Georgia

Owens, Leigh

Oyama, Fumitaka

Ozaki, Iwata

Ozaki, Toshinori

Ozawa, Koichiro

Ozeki, Yasuhiro

Ozeki, Yoshihiro

Pace, Vittorio

Padhye, Nikhil

Padilla, Daniel

Padmalayam, Indira

Padmanabhan, Jaya

Padula, Matthew

Paeshuyse, Jan

Pagan, One
Yoneyama, Tadakatsu

Yoo, Dongwan

Yoo, Eun-Sook

Yoo, Wan-Hee

Yoon, Do-Young

Yoon, Hwan Su

Yoon, Jaeseung

Yoon, Je-Hyun

Yoon, Minjoong

Yoon, Moon-Young

Yoon, Seog-young

Yoon, Shin H.

Yoshida, Hiderou

Yoshida, Hiroshi

Yoshida, Kunihiro

Yoshii, Toshitaka

Yoshikawa, Masaaki

Yoshimitsu, Takehiko

Yoshimura, Akihiko

Yoshimura, Kazuya

Yoshimura, Koichi

Yoshioka, Hideyuki

Yoshitomi, Hiroyuki

You, Liang

Young, Robert S.

Yoza, Brandon A.

Yperman, Jan

Yu, Cheng-Chia

Yu, Cheng-Ping

Yu, Chia-Li

Yu, Chin-sheng

$\mathrm{Yu}$, Fengqun

$\mathrm{Yu}$, Jae-Ran

$\mathrm{Yu}$, Jennifer S.

Yu, Lijian

Yu, Mengxiao

Yu, Yan Ping

Yu, Yongjun

Yu, Yongxin Jeremy

Yuan, Xiao

Yuasa, S.

Yue, PatrickYingKit

Yuen, Tony

Yuh, Chiou-Hwa

Yull, Fiona E.

Yun, Dai

Yun, Hae Keun

Yun, Jong Won

Yunus, Agha

Yusupova, Gulnara

Zabel, Brian A.

Zabetakis, Ioannis

Zablackis, Earl 
Hatae, Noriyuki

Hatakeyama, Katsunori

Hati, Sanchita

Hatle, J.

Hauber, Joachim

Haughton, David

Havird, Justin C.

Hayakawa, Tohru

Hayes, Don, Jr.

Hayes, Maria

Hayes, Richard

Haynes, Cole M.

Hazlett, Linda D.

$\mathrm{He}$, Hong

He, Quan Sophia

He, Songbing

Headon, Denis J.

Hearing, Patrick

Heath, P.R.

Hebbard, L.

Hedhammar, My

Hedley, Peter

Heegaard, Niels H.H.

Hefferon, Kathleen Laura

Helariutta, Yrjö

Helder, Marco

Heldring, Nina

Hellweg, Christine E.

Henao-Martinez, Andres F.

Hendricks, Theo

Hendrickson, C.L.

Hendriksen, Peter J.m.

Hennebelle, Thierry

Hennies, Hans Christian

Hennig, Frauke

Henrich, Birgit

Henrich, Curtis J.

Henry, Morgane

Hera, Concha

Herath, Tharangani

Herbert, Karl Herbert

Heredia, Unai López

Herington, Jennifer L.

Hermans, Emmanuel

Hernández, J.A.

Hernandez, Pilar

Hernandez-Ledesma, B.

Herndon, David

Herranz, Raul Cano

Herrmann, Andreas

Herrmann, Martin

Herse, Florian

Herst, Patries
Paik, Jisun

Pal, Rituraj

Palazzetti, Federico

Palazzo, Alexander F.

Palenzuela López, José A.

Pallauf, Kathrin

Pallavicini, Alberto

Palm, Gottfried J.

Palm, Thomas

Palumbo, Carla

Pan, Chun-Hsu

Pan, Min-Hsiung

Panaro, Maria Antonietta

Panda, Amaresh C.

Pañeda, Covadonga

Panfoli, Isabella

Pang, Yi

Panico, Antonio

Pantano, Patrizia

Pantos, Constantinos

Pantoș, G. Dan

Panzella, Lucia

Paolocci, Nazareno

Papaccio, GianPaolo

Papagerakis, Petros

Papait, Roberto

Papassiopi, Nymphodora

Papathanos, Philippos

Papi, Alessio

Paradisi, Francesca

Parahuleva, Mariana S.

Pardo, Maria

Parenti, Serena Incerti

Parini, Rossella

Park, Chail-Il

Park, Enoch

Park, Eugene

Park, Hun-kuk

Park, In-Kyu

Park, Jeong-sook

Park, Jong-Chul

Park, Jong-Hoon

Park, Ky Young

Park, Sunghyouk

Park, Tae Jung

Park, Woo Hyun

Park, Yongsoon

Parker, Anthony W.

Parks, Robin

Parnell, Jill A.

Parniakov, Oleksii

Parodis, Ioannis

Parrella, Paola
Zaccone, Claudio

Zachara, Natasha E.

Zachut, Maya

Zago, Miriam

Zagouri, Flora

Zahid, Maliha

Zakeri, Bijan

Zakhartchouk, Alexander

Zaknun, John J.

Zalapa, Juan Ernesto

Zamaratskaia, Galia

Zan, Jindong

Zanke, Brent

Zannad, Faiez

Zannas, Anthony S.

Zannoni, Gian Franco

Zanotti, Giuseppe

Zaravinos, Apostolos

Zarbock, Ralf

Zarogoulidis, Paul

Žárský, Viktor

Zavitsas, Andreas

Zehbe, Rolf

Zeilinger, Carsten

Zelarayán, Laura C.

Zenarruzabeitia, Olatz

Zeng, Huaqiang

Zeng, Lingfang

Zeng, Xin-Xin

Zerbini, Gianpaolo

Zervos, Antonis S.

Zgaga, Lina

Zgaljardic, Dennis J.

Zhang, Caiguo

Zhang, Can

Zhang, Chengfei

Zhang, Haoqian

Zhang, Qi

Zhang, Qing

Zhang, Shaoping

Zhang, Tuo

Zhang, Xuchen

Zhang, Xuehong

Zhang, Xun

Zhang, Yanjin

Zhang, Yong

Zhang, Zhenhua

Zhao, Liang

Zhao, Shou-Jing

Zhao, Youfu

Zhbannikov, Ilya Y.

Zheng, Guoping

Zheng, Wenguang 
Herzog, Fabian

Hesser, Hugo

Hettich, Robert

Heuberger, Adam

Heyd, Florian

Heymans, Stephane

Heyninck, Karen

Hiblot, Julien

Higgins, Paul J.

Highley, John Robin

Higuchi, Akon

Hili, Ryan

Hill, Jeff W.

Hiltermann, T. Jeroen $\mathrm{N}$.

Himeno, Seichiro

Hinds, Terry D., Jr.

Hinkel, Rabea

Hinsen, Konrad

Hinton, Cimona

Hirahara, Kiyoshi

Hirasawa, Noriyasu

Hirashima, Akinori

Hirayama, Fumitoshi

Hiroaki, Fujii

Hirsch, Anna K.H.

Hirsch, Rhoda Elison

Hisabori, Toru

Hjalmarsson, Clara

Ho, Michael (Shan-Hui)

Ho, Vikki

Ho, Wen-Fu

Hocher, Valerie

Hodgson, Louis

Hodson, Leanne

Hoemann, Caroline D.

Hoffjan, Sabine
Parrish, Alan

Parsonage, Derek

Parsons, Barry J.

Pasán, Jorge

Pascault, Jean-pierre

Pascual-Teresa, Sonia

Pasini, Dario

Pasini, Luigi

Pask, Andrew J.

Passi, Alberto

Pastor, Isabel

Pastor, Jesús

Patarrão, Rita

Pater, Sylvia de

Pathak, Harsh

Patil, Ashwini

Patriarca, Carlo

Pattenden, Gerald

Patti, Francesco

Pattison, David I.

Paulmurugan, Ramasamy

Paulsen, Berit

Paulson, Elizabeth L.

Pavel, Ioana E.

Pavert, Steven H. P. van de

Pavlov, Youri I.

Pavon-Djavid, Graciela

Pavone, Luigi Michele

Pawlak, Rémy

Pazzola, Michele

Pearson, James T.

Pedeux, Rémy

Peijnenburg, Willie J.G.M.

Peker, Deniz

Pekkinen, Minna
Zhong, Xue

Zhou, Beiyan

Zhou, Bing

Zhou, Binhua P.

Zhou, Daohong

Zhou, Heling

Zhou, Huan

Zhou, Man

Zhou, Qingxiang

Zhou, Weijun

Zhou, Wenguang

Zhou, Xiangrong

Zhu, Changfu

Zhu, Meng-Yang

Zhu, Qian-Hao

Zhu, Xiaoshan

Zhu, Yefei

Zhuang, Jie

Ziai, Wendy C.

Zignego, Anna Linda

Zinzen, Robert

Ziparo, Elio

Zivadinov, Robert

Zodrow, Erwin L.

Zolotukhin, Sergei

Zorzano, Antonio

Zou, Shengli

Zou, Xueqing

Zsurka, Gabor

Zucman-Rossi, Jessica

Zullo, Letizia

Zuo, Yuegang

Zuppinger, Christian

Zwart, Mark P.

Zwaveling, J. 